\title{
Identification of androgen-responsive IncRNAs as diagnostic and prognostic markers for prostate cancer
}

\author{
Xuechao Wan ${ }^{1, *}$, Wenhua Huang ${ }^{1, *}$, Shu Yang ${ }^{1}$, Yalong Zhang ${ }^{1}$, Honglei Pu${ }^{1}$, Fangqiu \\ $\mathbf{F u}^{1}$, Yan Huang ${ }^{1}$, Hai Wu ${ }^{1}$, Tao $\mathbf{L i}^{3}$, Yao $\mathbf{L i}^{1,2}$ \\ ${ }^{1}$ State Key Laboratory of Genetic Engineering, Shanghai Engineering Research Center Of Industrial Microorganisms, School \\ of Life Science, Fudan University, Shanghai 200433, PR China \\ ${ }^{2}$ Key Laboratory of Reproduction Regulation of NPFPC, Fudan University, Shanghai 200433, PR China \\ ${ }^{3}$ Department of Urology, Tongji Hospital, Tongji University School of Medicine, Shanghai 200433, PR China \\ *These authors contributed equally to this work \\ Correspondence to: Yao Li, email: yaoli@fudan.edu.cn \\ Tao Li, email: quicktao@sina.com \\ Keywords: long non-coding RNA, prostate cancer, androgen receptor, expression profiling, biomarkers \\ Received: September 24, $2015 \quad$ Accepted: July 26, $2016 \quad$ Published: August 19, 2016
}

\section{ABSTRACT}

Prostate cancer ( $\mathrm{PCa}$ ) is a leading cause of mortality among males. Long non-coding RNAs (IncRNAs) are subclass of noncoding RNAs that may act as biomarkers and therapeutic targets. In this study, we firstly conducted analysis of global IncRNA expression patterns by using our own cohort (GSE73397) and two public available gene expression datasets: The Cancer Genome Atlas (TCGA) and GSE55909. Next, we performed microarray to observe genome-wide IncRNAs' expressions under dihydrotestosterone (DHT) stimulation in LNCaP cells (GSE72866), and overlapped the result with ChIPBase data to predict androgen-responsive IncRNAs with ARE. Combined the two results, a total of 44 androgen-responsive IncRNAs with ARE were found to be over-expressed in PCa samples. Ten IncRNAs were selected for further validation by examining their expressions in LNCaP cells under DHT stimulation, and in PCa samples and cell lines. Among them, RP1-4514.2, LINC01138, SUZ12P1 and KLKP1 were validated as directly AR-targeted IncRNAs by ChIP-PCR. Then we conducted a bioinformatic analysis to identify IncRNAs as putative prognostic and therapeutic targets by using TCGA data. Three androgen-responsive IncRNAs, LINC01138, SUZ12P1 and SNHG1 showed association with gleason score and pT-stage. The biological functions of LINC01138 and SUZ12P1 were also evaluated, both IncRNAs promoted the proliferation and inhibited apoptosis of $\mathrm{PCa}$. These results provide potent information for exploring potential biomarkers and therapeutic targets for prostate cancer, especially for castration-resistant PCa.

\section{INTRODUCTION}

Long noncoding RNAs (lncRNAs) are RNA polymerase II (RNAPII) transcripts of more than 200 nucleotides with no protein coding function [1]. Increasingly reports show that lncRNAs play important roles in tumorigenesis, cancer progression, and metastasis by regulating expression of protein-coding genes through transcriptional, post-transcriptional, post-translational and/or epigenetic regulation [2-5]. For example, lncRNA CTBP1-AS, associates with PSF, recruits the HDACSin3A complexes to CTBP1 promoter in cis and also guides PSF complexes to the regulatory regions of their endogenous target genes in trans-regulatory pathway [6]. LncRNAs play different roles in various cancers, either oncogenes (PCGEM1, CTBP1-AS) or tumor suppressor genes (GAS5, LincRNA-p21 and PCAT29) [7-10].

Prostate cancer $(\mathrm{PCa})$ is the most commonly diagnosed cancer of men in the United States with secondly leading cause of death in 2015[11]. Androgen receptor (AR) play a pivotal role in prostate cancer $(\mathrm{PCa})$ development at all stages, including the "androgenindependent" tumors [12]. Androgen ablation is a primary therapy of prostate cancer. However, after 18 to 24 months of androgen deprivation, $\mathrm{PCa}$ will eventually progress to castration-resistant form with limited therapeutic options 
and poor prognosis [13-15]. The accurate molecular mechanisms for hormone independent behavior of $\mathrm{PCa}$ remain unclear. Recently, emerging evidence suggests a few AR-regulated lncRNAs including CTBP1-AS, PCAT18 and PCAT29 show association with PCa androgen-resistance transformation $[6,16,17]$. Thus, we attempted to systematically identify androgen-responsive lncRNAs that may mediate the processes from "androgendependent" to "androgen-independent" in PCa.

On the other hand, risk stratification of $\mathrm{PCa}$ patients at diagnosis is mainly dependent on prostate-specific antigen (PSA) testing, which has low specificity for cancer distinguishing from $\mathrm{PBH}$, and cannot discriminate between indolent and metastatic castration-resistant PCa (mCRPC) $[18,19]$. Therefore, there is a significant need to identify potential novel prognostic biomarkers. Several previously characterized lncRNAs (SChLAP1, PCGEM1 and PCA3) show disease-associated overexpression [20-22]. Notably, PCA3 showed better performance than PSA in urinary detection to detect PCa [23]. Unfortunately, PCA3 still couldn't be able to discriminate between indolent and clinically aggressive PCa [24].

In this study, we firstly conducted analysis of global lncRNA expression patterns by using our own cohort (GSE73397) and two public available gene expression datasets: The Cancer Genome Atlas (TCGA) and GSE55909. Next, we performed microarray to observe genome-wide lncRNAs' expressions under dihydrotestosterone (DHT) stimulation in LNCaP cells (GSE72866). Then we conduct a bioinformatic analysis to identify lncRNAs as putative prognostic and therapeutic targets by using TCGA data. Our results suggest that the lncRNAs we report may provide new molecular biomarkers for the diagnosis of prostate cancer, especially for castration-resistant $\mathrm{PCa}$.

\section{RESULTS}

\section{Identification of differentially expressed androgen-responsive IncRNAs in prostate cancer patients}

Global expressions of lncRNAs in PCa samples and normal prostate tissues were examined by RiboArray ${ }^{\mathrm{TM}}$ Custom Array (Arraystar, Rockville, MD) and 982 lncRNAs with differential expression $(p<0.1)$ between PCa samples and normal prostate tissues were selected for further analysis. Of the lncRNAs, 501 lncRNA transcripts produced from 467 genes were up-regulated and 481 transcripts produced from 443 genes were down-regulated in prostate cancer. The list of the top 30 differentially expressed lncRNAs identified by microarray analysis was shown in Table 1. Hierarchical clustering of the differentially expressed lncRNAs was shown in Figure 1A.

To maximize the coverage of lncRNA expression profiling, another two publicly available gene expression data, GSE55909 and TCGA, were also used to identify the differentially expressed lncRNAs in prostate cancer. We firstly analyzed GSE55909 database and identified 318 lncRNAs produced from 250 genes were up-regulated and 1927 lncRNAs produced from 1653 genes were down-regulated in prostate cancer compared with normal tissues. Hierarchical clustering of the differentially expressed lncRNAs was shown in Figure 1B. Then, RNAseq data from TCGA, a cohort of 52 normal prostate tissues and 419 prostate cancer samples, were analyzed. According to OE Human lncRNA Microarray V2.0, which contains 4321 lncRNAs with description from NCBI, there are 1410 lncRNAs that can be found in TCGA dataset. After filtering the dataset to remove the lncRNAs that have low expression levels, we ended up with 654 expressed lncRNAs that are potentially relevant in prostate cancer (Supplementary Table S3). Compared with the normal prostate tissues, we found that there were 252 lncRNAs that were upregulated $(p<0.05)$ and there were 157 lncRNAs that were down-regulated $(p<0.05)$ in prostate cancer. Hierarchical clustering of the differentially expressed lncRNAs was shown in Figure 1C.

To identify androgen-responsive lncRNAs, Agilent Human lncRNA array was performed to simultaneously observe lncRNAs and mRNAs expressions in androgendependent LNCaP cells under DHT stimulation in time points of $0 \mathrm{~h}$ and $2 \mathrm{~h}$, respectively. The ' $0 \mathrm{~h}$ ' worked as the control representing cellular status before DHT stimulation. Supervised analysis of the microarray data showed 3767 deregulated lncRNA transcripts (1991 transcripts up-regulated and 1776 transcripts down-regulated) produced from 2980 genes, with an average expression level over 2-fold change in $2 \mathrm{~h}$ compared to $0 \mathrm{~h}$ (GSE72866). The list of the top 15 upregulated and top 15 downregulated transcripts identified by microarray analysis was shown in Table 2 .

Noteworthy, being androgen-responsive lncRNAs does not mean being directly regulated by activated AR. Our major aim was to pick out AR directly regulated lncRNAs, which may play important roles in mediating AR signaling and $\mathrm{PCa}$ androgen-resistance transformation. ChIPBase data [25] was used and showed 312 androgen-responsive lncRNAs (including $10 \mathrm{~kb}$ up- and $1 \mathrm{~kb}$ downstream region of lncRNAs' transcription start site) overlapping AR peaks (Supplementary Figure S1; Supplementary Tables S1, S2) may be directly regulated by AR.

To identify androgen-responsive lncRNAs as diagnostic and prognostic markers for prostate cancer, we performed integrated analysis of differentially expressed lncRNAs and androgen-responsive lncRNAs in $\mathrm{PCa}$ patients. A total of 44 androgen-responsive lncRNAs were found to be over-expressed and 95 lncRNAs were found to be down-regulated in PCa samples (Figure 1D and 1E). We are primarily focusing on the up-regulated lncRNAs as putative biomarkers in prostate tumor compared with normal tissues. Of 44 up-regulated androgenresponsive lncRNAs, 3 androgen-reduced lncRNAs and 7 
Table 1: Top 30 differentially expressed IncRNAs in prostate cancer samples identified by microarray analysis

\begin{tabular}{|c|c|c|c|c|c|c|c|c|c|c|c|}
\hline ProbeName & Gene Symbol & $\begin{array}{c}\text { FC } \\
(\text { abs) }\end{array}$ & pval & Regulation & T1 & $\mathbf{T 2}$ & T3 & N1 & $\mathbf{N 2}$ & $\mathbf{N 3}$ & Chr \\
\hline ENST00000584428 & RP5-890E16.2 & 3.92 & 0.04 & down & 10.74 & 11.07 & 9.16 & 11.82 & 12.87 & 12.19 & Chr 17 \\
\hline ENST00000572222 & MMP25-AS1 & 3.32 & 0.05 & down & 9.50 & 10.81 & 8.96 & 11.74 & 10.94 & 11.78 & Chr 16 \\
\hline ENST00000425192 & AC073641.2 & 3.09 & 0.02 & down & 8.52 & 7.82 & 7.57 & 8.97 & 10.17 & 9.66 & Chr 2 \\
\hline ENST00000582858 & RP11-192H23.8 & 2.64 & 0.04 & down & 9.88 & 10.18 & 9.51 & 11.95 & 11.39 & 10.43 & Chr 17 \\
\hline ENST00000517345 & RP11-30J20.1 & 2.48 & 0.06 & down & 8.78 & 7.63 & 7.68 & 8.65 & 9.61 & 9.75 & Chr 8 \\
\hline ENST00000432195 & RP4-575N6.4 & 2.34 & 0.04 & down & 7.68 & 8.34 & 7.10 & 9.18 & 9.04 & 8.57 & Chr 1 \\
\hline ENST00000414668 & LINC00029 & 2.30 & 0.10 & down & 7.96 & 9.33 & 9.48 & 9.62 & 10.61 & 10.16 & Chr 20 \\
\hline ENST00000537370 & CCND2-AS2 & 2.30 & 0.07 & up & 9.11 & 10.55 & 9.11 & 8.11 & 8.59 & 8.46 & Chr 12 \\
\hline ENST00000523445 & TPT1-AS1 & 2.25 & 0.07 & down & 8.03 & 9.41 & 8.03 & 9.42 & 9.92 & 9.65 & Chr 13 \\
\hline ENST00000491849 & LINC00971 & 2.19 & 0.03 & up & 9.58 & 10.49 & 9.86 & 8.57 & 8.69 & 9.28 & Chr 3 \\
\hline ENST00000366437 & MIR205HG & 2.19 & 0.02 & down & 9.10 & 9.33 & 8.68 & 9.80 & 10.52 & 10.18 & Chr 1 \\
\hline ENST00000443892 & RP3-395M20.8 & 2.12 & 0.01 & down & 10.14 & 10.26 & 10.15 & 11.66 & 11.38 & 10.78 & Chr 1 \\
\hline ENST00000530223 & RP5-1047A19.4 & 2.07 & 0.08 & up & 11.41 & 10.29 & 9.99 & 9.38 & 9.49 & 9.67 & Chr 8 \\
\hline ENST00000577848 & RP11-874J12.4 & 2.01 & 0.05 & down & 8.80 & 9.40 & 8.50 & 9.49 & 9.87 & 10.36 & Chr 18 \\
\hline ENST00000426364 & LINC00633 & 2.01 & 0.03 & up & 10.46 & 9.45 & 9.88 & 8.90 & 9.00 & 8.88 & Chr X \\
\hline ENST00000577560 & CTD-2006K23.1 & 1.98 & 0.10 & down & 8.48 & 9.03 & 7.89 & 10.09 & 9.26 & 9.01 & Chr 17 \\
\hline ENST00000519967 & RP11-705O24.1 & 1.97 & 0.09 & up & 10.40 & 9.93 & 10.05 & 8.87 & 8.64 & 9.95 & Chr 8 \\
\hline ENST00000568635 & CTA-363E6.5 & 1.95 & 0.04 & up & 9.60 & 10.32 & 9.64 & 8.88 & 8.51 & 9.29 & Chr 16 \\
\hline ENST00000455238 & RP11-193P11.3 & 1.94 & 0.04 & down & 9.34 & 9.82 & 8.69 & 10.19 & 10.30 & 10.22 & Chr 1 \\
\hline ENST00000555070 & RP11-33N16.3 & 1.94 & 0.01 & up & 9.54 & 10.03 & 9.84 & 8.74 & 8.68 & 9.14 & Chr 14 \\
\hline ENST00000554532 & AC005519.4 & 1.92 & 0.10 & down & 8.97 & 9.29 & 7.87 & 9.74 & 9.72 & 9.49 & Chr 14 \\
\hline ENST00000557989 & RP11-680F8.3 & 1.91 & 0.02 & down & 9.00 & 8.52 & 8.33 & 9.80 & 9.56 & 9.30 & Chr 15 \\
\hline ENST00000413063 & WASF3-AS1 & 1.89 & 0.03 & up & 9.64 & 10.17 & 9.29 & 8.69 & 8.96 & 8.68 & Chr 13 \\
\hline ENST00000564455 & AC009120.5 & 1.89 & 0.10 & down & 8.88 & 10.03 & 8.75 & 10.38 & 10.02 & 10.01 & Chr 16 \\
\hline ENST00000544717 & RP11-319E16.1 & 1.89 & 0.10 & up & 9.84 & 10.24 & 9.81 & 8.26 & 9.52 & 9.37 & Chr 12 \\
\hline ENST00000579057 & LINC00271 & 1.88 & 0.10 & up & 8.98 & 9.84 & 9.63 & 8.11 & 8.39 & 9.22 & Chr 6 \\
\hline ENST00000455894 & LINC00378 & 1.88 & 0.06 & up & 10.11 & 10.38 & 9.96 & 8.63 & 9.37 & 9.72 & Chr 13 \\
\hline ENST00000513893 & LINC00461 & 1.87 & 0.08 & down & 8.72 & 9.57 & 9.05 & 10.05 & 9.50 & 10.50 & Chr 5 \\
\hline ENST00000537095 & FZD10-AS1 & 1.86 & 0.03 & up & 9.98 & 10.42 & 9.91 & 9.08 & 8.91 & 9.62 & Chr 12 \\
\hline ENST00000568707 & CTD-2323K 18.1 & 1.86 & 0.02 & up & 9.59 & 9.43 & 9.46 & 8.84 & 8.84 & 8.11 & Chr 15 \\
\hline
\end{tabular}

T-Tumor.

N-Normal.

androgen-induced lncRNAs were randomly selected for further study.

\section{Validation of androgen-responsive IncRNAs using qRT-PCR}

To further confirm the time-course microarray data, 10 lncRNAs (RP1-4514.2, SUZ12P1, SNHG5, LINC01138, SNHG1, KLKP1, LINC00969, LINC-PINT, TUG1 and MIR17HG) were selected for further qRT-PCR validation. The expression level of these lncRNAs from Agilent microarray data were shown in Figure 2A.

We firstly performed qRT-PCR analysis to observe lncRNAs expressions in $\mathrm{LNCaP}$ cells under DHT stimulation in a time series of $0 \mathrm{~h}, 2 \mathrm{~h}, 8 \mathrm{~h}, 24 \mathrm{~h}$ and $48 \mathrm{~h}$, respectively. Five lncRNAs, RP1-4514.2, SUZ12P1, SNHG5, LINC01138, and SNHG1, were down-regulated under DHT treatment (Figure 2B-2F). Five IncRNAs, KLKP1, LINC00969, LINC-PINT, TUG1 and MIR17HG, were up-regulated under DHT treatment (Figure 2G-2K). Notably, RP1-4514.2 and LINC01138 were induced and KLKP1 was reduced over 2 fold after AR silenced, suggesting the involvement of AR in androgen-mediated regulation of these lncRNAs expressions. Puzzlingly, these results are not completely consistent with our microarray data.

Considering the limited time points and high falsepositive rate of microarray, a dose-response study was recruited to explore the expression of these lncRNAs under different doses of DHT $(0,0.1,1,10,100$ and $1000 \mathrm{nM})$. The results showed that these lncRNAs had the same expression tendency under DHT stimulation in a time series and in a dose series (Figure 3A-3J). In addition, the expression of 
LINC00308, the most up-regulated lncRNA in our data, in LNCaP cells treated with DHT in dose or time dependent study were also analyzed by qRT-PCR (Supplementary Figure S3A-S3B). The expression of androgen-responsive genes PSA (KLK3) and TMPRSS2 were used as positive controls (Supplementary Figure S2A-S2D).

\section{Verification the regulation of $A R$ on androgen- responsive IncRNAs}

To further validate the regulation of $\mathrm{AR}$ on lncRNAs, we performed siRNA-directed knockdown of AR in LNCaP cells. As shown in Figure 4A, siAR-544 remarkably decreased AR both mRNA and protein levels in LNCaP after siRNA transfection.

As our results shown, the expressions of five androgen-reduced lncRNAs, RP1-4514.2, SUZ12P1, SNHG5, LINC01138, and SNHG1, were significantly up-regulated after AR knockdown, the expressions of five androgen-induced lncRNAs, KLKP1, LINC00969, LINC-PINT, TUG1 and MIR17HG, were significantly down-regulated after AR knockdown (Figure 4B). The expression of androgen-responsive genes PSA (KLK3) and TMPRSS2 were used as positive controls.

RP1-4514.2, LINC01138, and SUZ12P1, the top three up-regulated lncRNAs, and KLKP1, the most down-regulated lncRNA after AR knockdown, were selected to validate the direct binding of AR to androgen response elements (AREs). The Genomatix database [26] was employed to predict potential androgen response elements (AREs) in the upstream $10 \mathrm{~kb}$ of the four lncRNAs transcription start site (TSS) (Figure 4C). AREs with 'Core Similarity $=1$ ', which represent the highest match between target DNA sequence and ARE's conserved bases, were chosen for further validation by ChIP. As the results, compared with control, androgen-

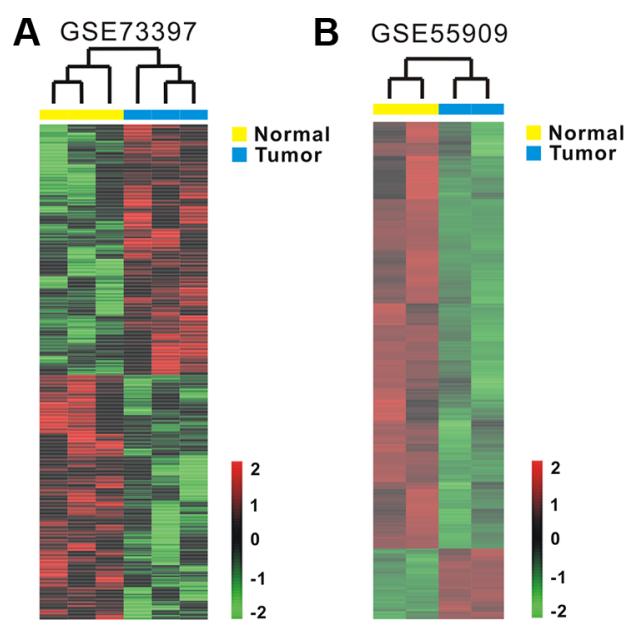

C TCGA
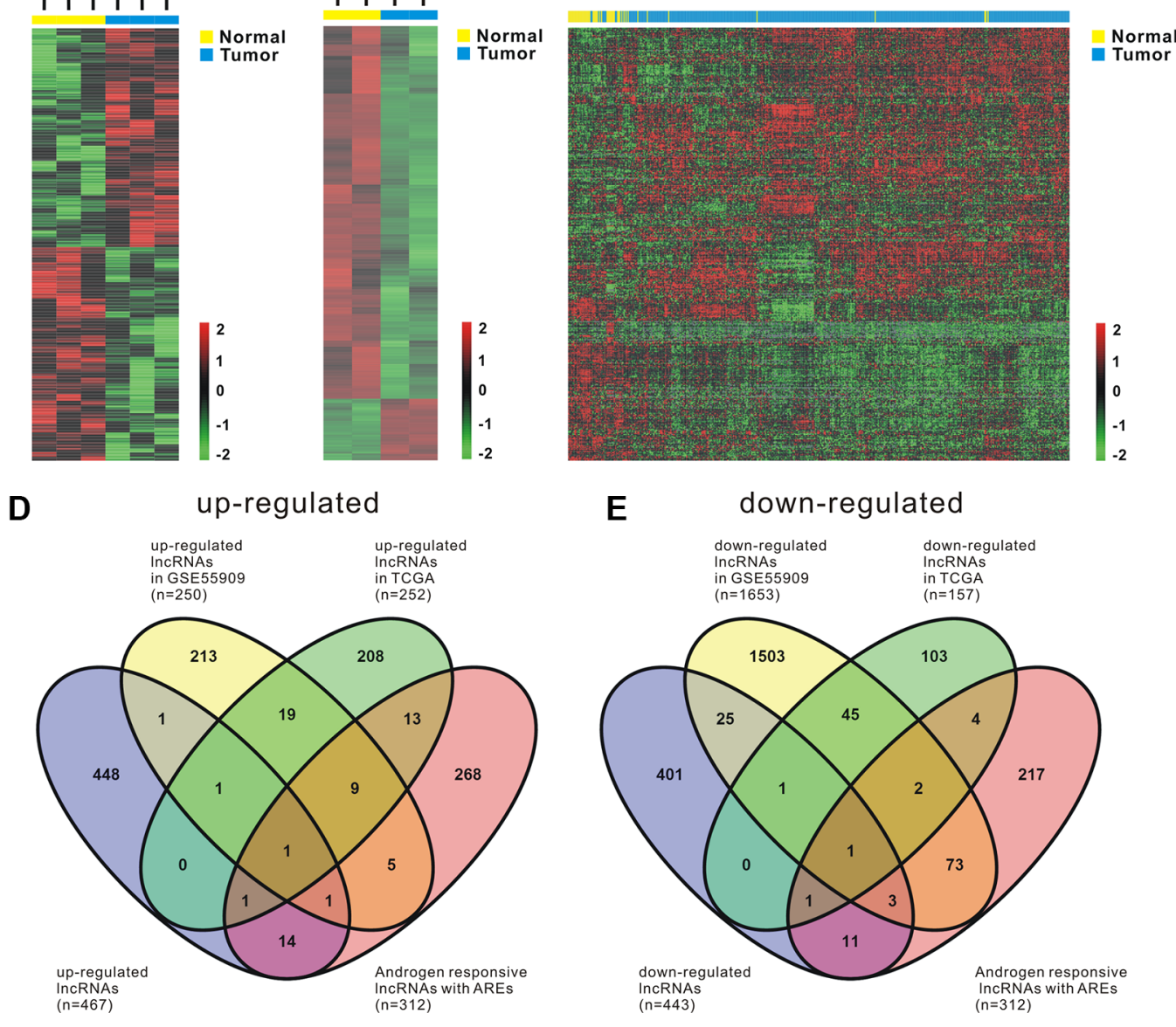

Figure 1: Comprehensive analysis of differentially expressed androgen-responsive IncRNAs in PCa patients. Heat map shows differential lncRNA expression between prostate tumor samples and normal tissues by using our own cohort (GSE73397) (A) and two publicly available gene expression data GSE55909 (B) and TCGA (C). Hierarchical clustering reveals distinguishable lncRNA expression profiles. Red indicates high relative expression and green indicates low relative expression. 2, 0 and -2 are folds changes in the corresponding spectrum, whereas Normal represents normal prostate samples and Tumor represents prostate cancer tissues. (D, E) four-way Venn diagrams display the overlap of up-regulated and down-regulated androgen-responsive lncRNAs that are in GSE73397, GSE55909, TCGA and GSE72866. 
Table 2: Top 30 differentially expressed transcripts under DHT stimulation identified by microarray analysis

\begin{tabular}{|c|c|c|c|c|c|c|c|}
\hline ProbeName & $\begin{array}{c}\text { Ensembl } \\
\text { Transcript ID }\end{array}$ & Gene Symbol & FC (abs) & Regulation & O hr & $2 \mathrm{hr}$ & Chr \\
\hline oebiotech_09219 & ENST00000419431 & LINC00308 & 47.02 & up & 3.61 & 9.16 & Chr 21 \\
\hline A_21_P0010982 & & MALAT1 & 41.79 & up & 8.17 & 13.55 & Chr 11 \\
\hline A_21_P0014694 & & LOC100509487 & 41.35 & up & 3.37 & 8.74 & Chr 16 \\
\hline oebiotech_21382 & ENST00000569100 & GOLGA8B & 38.43 & up & 4.13 & 9.39 & Chr 15 \\
\hline oebiotech_20175 & ENST00000507787 & HERC2P9 & 37.47 & up & 3.46 & 8.68 & Chr 15 \\
\hline A_19_P00331618 & & LOC100507645 & 37.41 & up & 8.88 & 14.11 & Chr 11 \\
\hline oebiotech_16752 & ENST00000425771 & GAS5 & 37.04 & up & 3.71 & 8.92 & Chr 1 \\
\hline oebiotech_17693 & ENST00000508832 & MALAT1 & 35.05 & up & 9.77 & 14.90 & Chr 11 \\
\hline A_33_P3291430 & ENST00000397750 & ST7-OT4 & 34.61 & up & 3.37 & 8.48 & Chr 7 \\
\hline oebiotech_05345 & ENST00000546959 & LINC01296 & 34.44 & up & 3.68 & 8.78 & Chr 14 \\
\hline oebiotech_20049 & ENST00000429124 & FTX & 30.87 & up & 3.40 & 8.35 & Chr X \\
\hline A_21_P0011477 & ENST00000440946 & AP000525.10 & 29.83 & up & 3.74 & 8.64 & Chr 14 \\
\hline A_21_P0010871 & ENST00000445221 & BMS1P1 & 29.65 & up & 4.80 & 9.69 & Chr 10 \\
\hline A_21_P0011370 & ENST00000360553 & GOLGA8A & 29.04 & up & 3.89 & 8.75 & Chr 15 \\
\hline A_19_P00319476 & & LOC100507645 & 28.89 & up & 8.63 & 13.49 & Chr 11 \\
\hline A_21_P0001069 & ENST00000416908 & RP11-449J1.1 & 32.29 & down & 8.45 & 3.44 & Chr 1 \\
\hline oebiotech_00606 & ENST00000419425 & AC006369.2 & 12.13 & down & 8.02 & 4.42 & Chr 2 \\
\hline A_21_P0002731 & ENST00000425195 & AC098973.2 & 11.22 & down & 7.07 & 3.58 & Chr 3 \\
\hline A_21_P0001785 & & FLJ33534 & 10.82 & down & 6.85 & 3.41 & Chr 2 \\
\hline A_21_P0011663 & & GTSCR1 & 10.06 & down & 7.52 & 4.19 & Chr 18 \\
\hline oebiotech_02576 & ENST00000455447 & TNR-IT1 & 9.09 & down & 6.58 & 3.39 & Chr 1 \\
\hline oebiotech_06568 & ENST00000561479 & RP11-586K12.4 & 8.85 & down & 6.73 & 3.58 & Chr 16 \\
\hline oebiotech_03425 & ENST00000507698 & RP11-438D8.2 & 8.62 & down & 6.68 & 3.57 & Chr 3 \\
\hline A_21_P0002701 & ENST00000429949 & LINC00690 & 8.53 & down & 6.46 & 3.37 & Chr 3 \\
\hline A_21_P0014499 & & SACS-AS1 & 8.01 & down & 7.20 & 4.20 & Chr 13 \\
\hline A_21_P0004212 & ENST00000512521 & RP11-121L11.3 & 7.53 & down & 7.50 & 4.59 & Chr 5 \\
\hline A_19_P00316241 & ENST00000504068 & RP11-434D9.1 & 7.38 & down & 6.37 & 3.48 & Chr 5 \\
\hline oebiotech_16555 & ENST00000413238 & LINC00689 & 7.17 & down & 6.33 & 3.49 & Chr 7 \\
\hline oebiotech_17270 & ENST00000460833 & ADAMTS9-AS2 & 7.02 & down & 6.21 & 3.40 & Chr 3 \\
\hline oebiotech_05235 & ENST00000543848 & RP11-7M8.2 & 6.84 & down & 6.55 & 3.78 & Chr 12 \\
\hline
\end{tabular}

activated AR was significantly recruited to the predicted AREs of RP1-4514.2, LINC01138, SUZ12P1, and KLKP1, in the presence of $100 \mathrm{nM}$ DHT in LNCaP cells for $4 \mathrm{~h}$ (Figure 4E). PSA enhancer (KLK3 promoter) and miR-125 b AREs were serves as the positive control for AR-binding, and XBP-1 promoter were serves as the negative control (Figure 4D). Collectively, all four IncRNAs are demonstrated as directly AR-targeted lncRNAs in androgen-dependent PCa.
Validation of differentially expressed IncRNAs in patient samples and PCa cell lines using qRT-PCR

We then validated the expression of differentially expressed lncRNAs in PCa patients to investigate their clinical value. The expressions of these ten lncRNAs in their corresponding databases were presented in Figure $5 \mathrm{~A}$. To further validate the results, we examined 
the expression levels in 11 normal prostate tissues and 14 prostate cancer tissues with real-time PCR (Figure 5B-5K). All of these lncRNAs except LINC00969, were expressed significantly higher in prostate cancer samples compared with normal prostate tissues, which were consistent with the TCGA and microarray results.

Then, the expression levels of ten IncRNAs were validated in the noncancerous prostatic cells WPMY-1 and four human prostate cancer cell lines 22RV1, DU145, PC-3 and LNCaP by qRT-PCR (Figure 6). It turned out that most of the ten lncRNAs were up-regulated in prostate cancer cell lines compared with WMPY-1 and showed a similar expression pattern in patient samples. In addition, the expression of CCND2-AS2, the most up-regulated lncRNA in our data, in tissue samples and prostate cancer cell lines were also analyzed by qRT-PCR (Supplementary Figure S3C-S3D).

\section{Androgen-responsive IncRNAs can serve as diagnostic markers in patient tissues}

As shown in Figure 1E, a total of 24 androgenresponsive lncRNAs were found to be up-regulated in TCGA. To evaluate possible prognostic value of these lncRNAs, we conducted a comprehensive bioinformatic
A

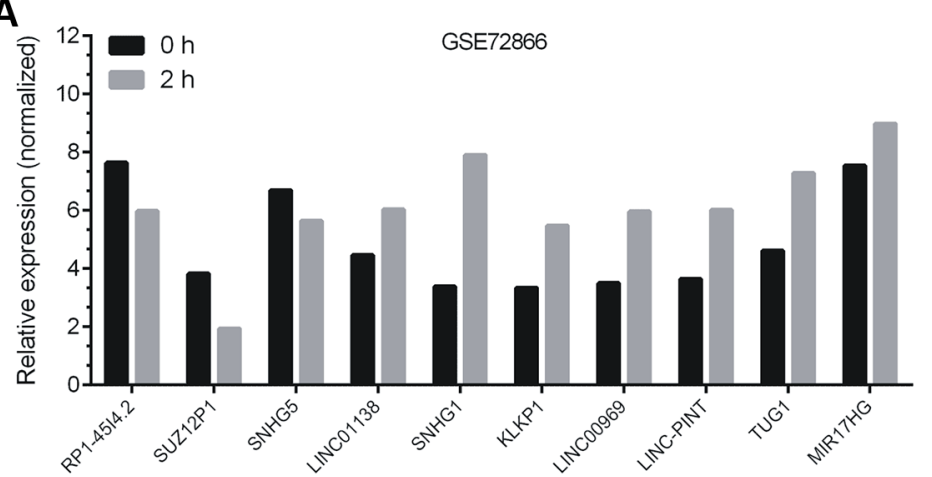

C

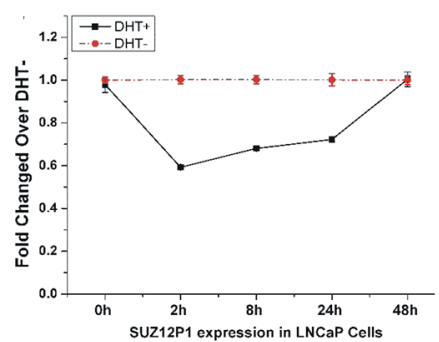

$\mathbf{F}$

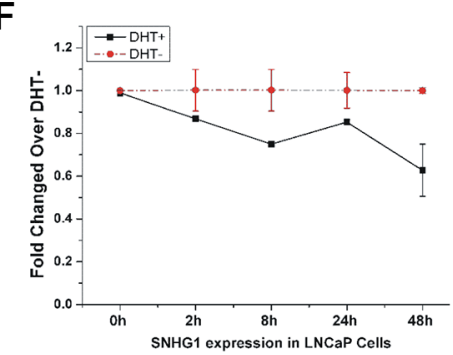

I

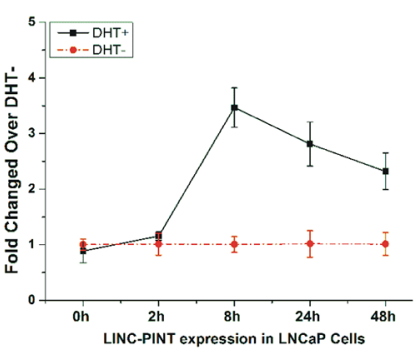

D

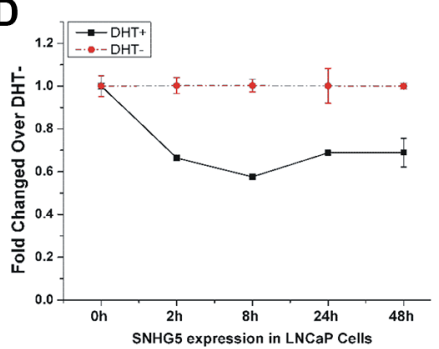

$\mathbf{G}$

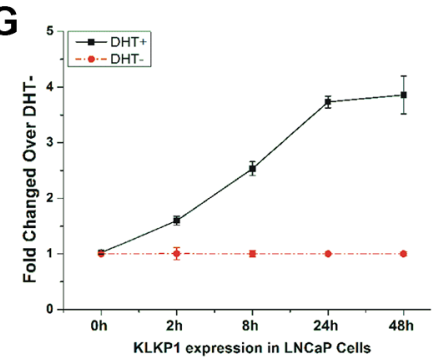

J

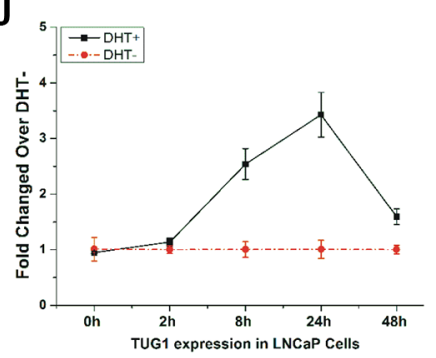

B

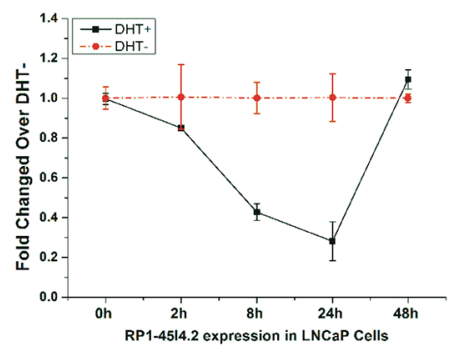

E

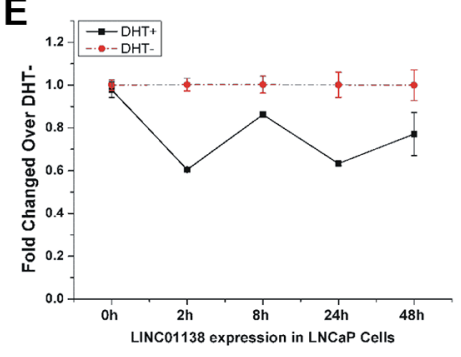

H

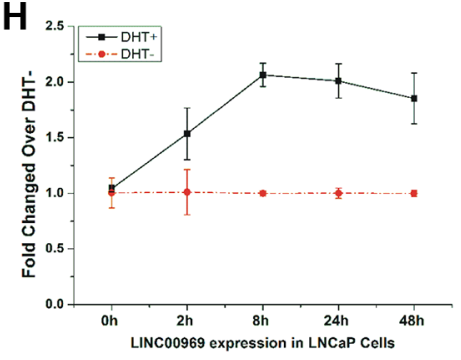

K

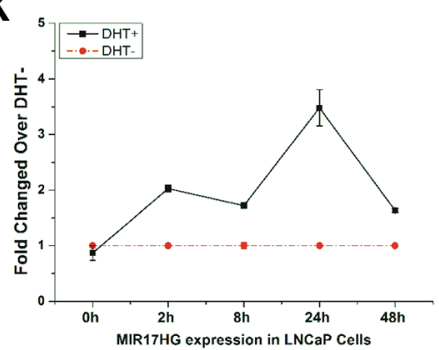

Figure 2: Validation of androgen-responsive IncRNAs in a time-dependent study. The expression level of candidate lncRNAs from Agilent microarray data were shown (A). (B-K) RT-PCR analyses of ten lncRNAs' expressions in LNCaP cells treated with $10 \mathrm{nM}$ DHT in time series of $0 \mathrm{~h}, 2 \mathrm{~h}, 8 \mathrm{~h}, 24 \mathrm{~h}$ and $48 \mathrm{~h}$. Values of expressions treated with equal volume of vehicle in the same time series were used as control. Results are presented as the means \pm s.d. of three independent experiments. 
analysis of TCGA database to identify the relationship between the expression of 24 lncRNAs and pathological grading and observed 3 androgen-responsive lncRNAs showed significant association with prostate cancer progression (Supplementary Tables S4, S5). As shown in Figure 7A-7F, LINC01138, SUZ12P1 and SNHG1 were expressed at lower levels with a low Gleason score $(\leq 7)$, compared with tumors with Gleason score $\geq 8$. Furthermore, the expression level of LINC01138, SUZ12P1 and SNHG1 markedly increased in invasive extraprostatic tumors (pT3a , pT3b and T4 stages) as compared with intraprostatic localized tumors (pT2a, pT2b and pT2c stages).

Next, we used a Kaplan-Meier analysis to evaluate whether 24 androgen-responsive lncRNAs expression were associated with patient outcome and 11 lncRNAs were found to be $(p<0.05)$. As expected, we found that a high level of LINC01138 $(p=0.0085)$, SUZ12P1 $(p=0.0031)$ and SNHG1 $(p=0.0009)$ were associated with
A

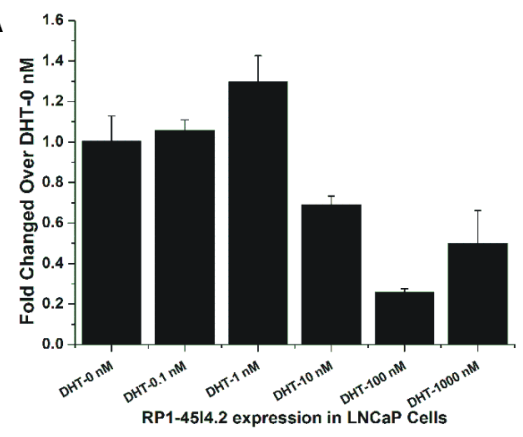

D

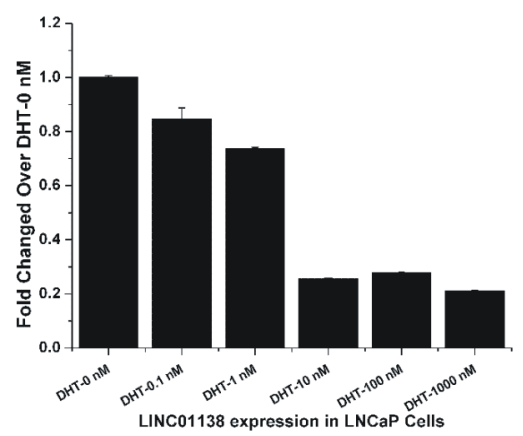

G

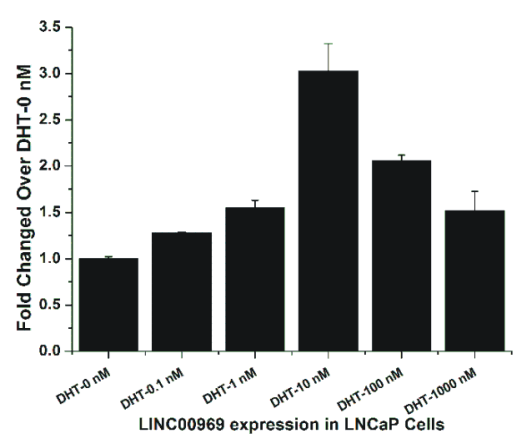

J

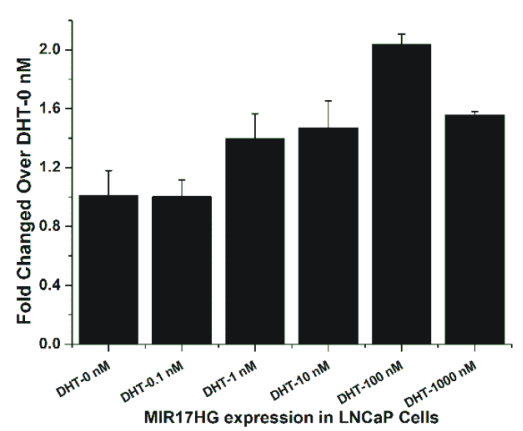

B

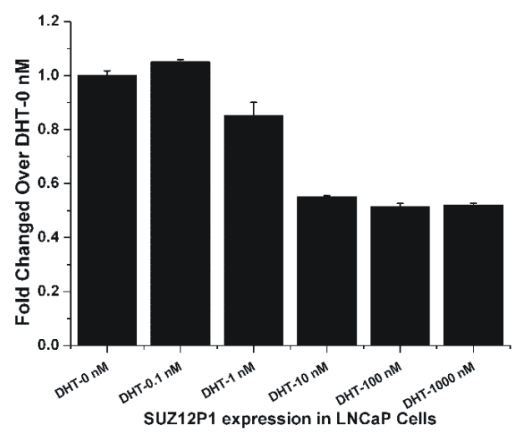

E

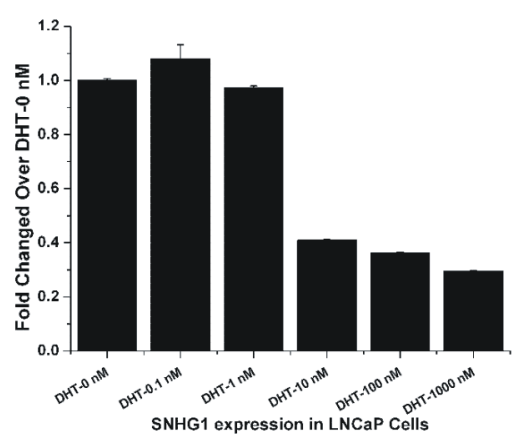

H

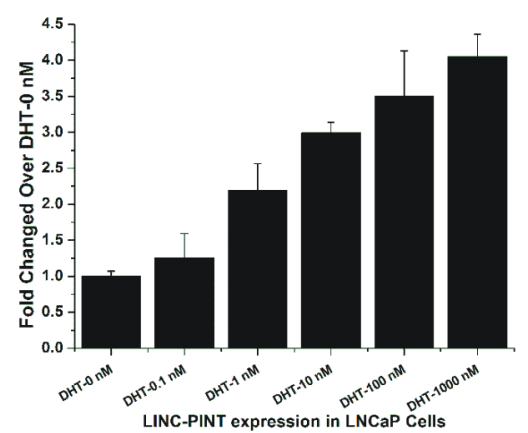

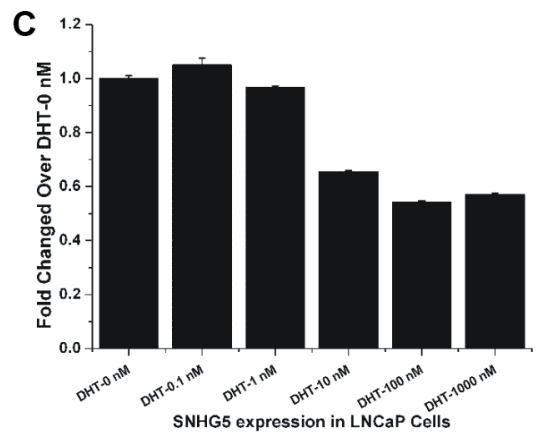
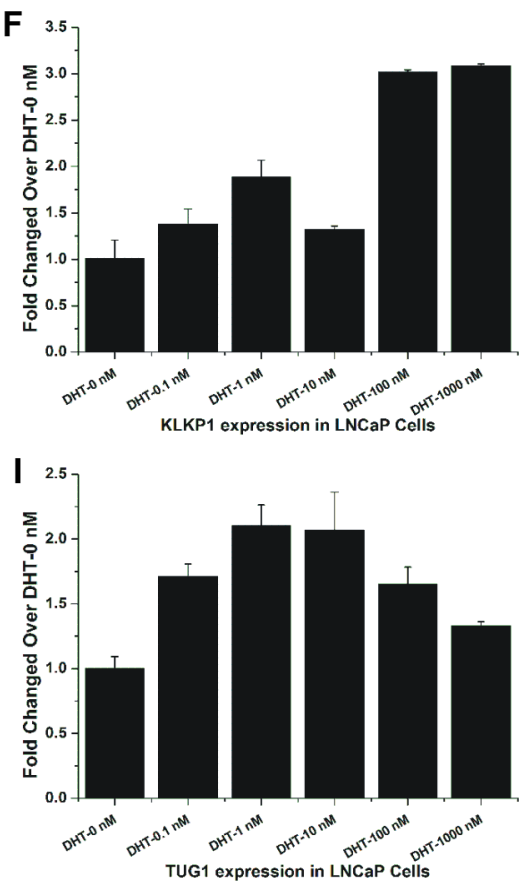

Figure 3: Validation of androgen-responsive IncRNAs in a dose-dependent study. (A-J) RT-PCR analyses of ten lncRNAs' expressions in LNCaP cells treated with DHT for $24 \mathrm{~h}$ in dose series of $0 \mathrm{nM}, 0.1 \mathrm{nM}, 1 \mathrm{nM}, 10 \mathrm{nM}, 100 \mathrm{nM}$ and $1000 \mathrm{nM}$. Values of dose zero were used as control. Results are presented as the means \pm s.d. of three independent experiments. 
significantly lower biochemical recurrence (Figure 7G-7I), indicating that the high level of LINC01138, SUZ12P1 and SNHG1 were correlated with a short biochemical recurrence-free survival times.

\section{LINC01138 and SUZ12P1 promoted the proliferation and inhibited apoptosis of PCa}

To evaluate the biological functions of these lncRNAs, we picked two lncRNAs, LINC01138 and SUZ12P1, and used small interfering RNA (siRNA) to down-regulate their expression in LNCaP cells. The
CCK8 assay showed that knockdown of LINC01138 or SUZ12P1 markedly suppressed cell viability in LNCaP cells (Figure 8A-8D and Supplementary Figure S4AS4B). We also observed that LINC01138 or SUZ12P1 knockdown in LNCaP and PC-3 cells significantly increased the proportion of cells in G1 phase and decreased that of cells in $\mathrm{S}$ phase by flow cytometry (Figure 8E-8F and Supplementary Figure S4C-S4F). We then explored the influence of LINC01138 and SUZ12P1 on apoptosis of prostate cancer cells lines using cell apoptosis assay, and found knockdown of them increased the fraction of apoptotic cells in

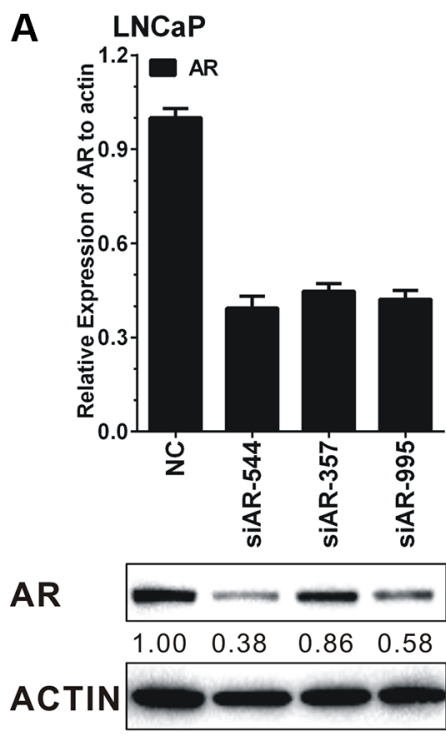

B
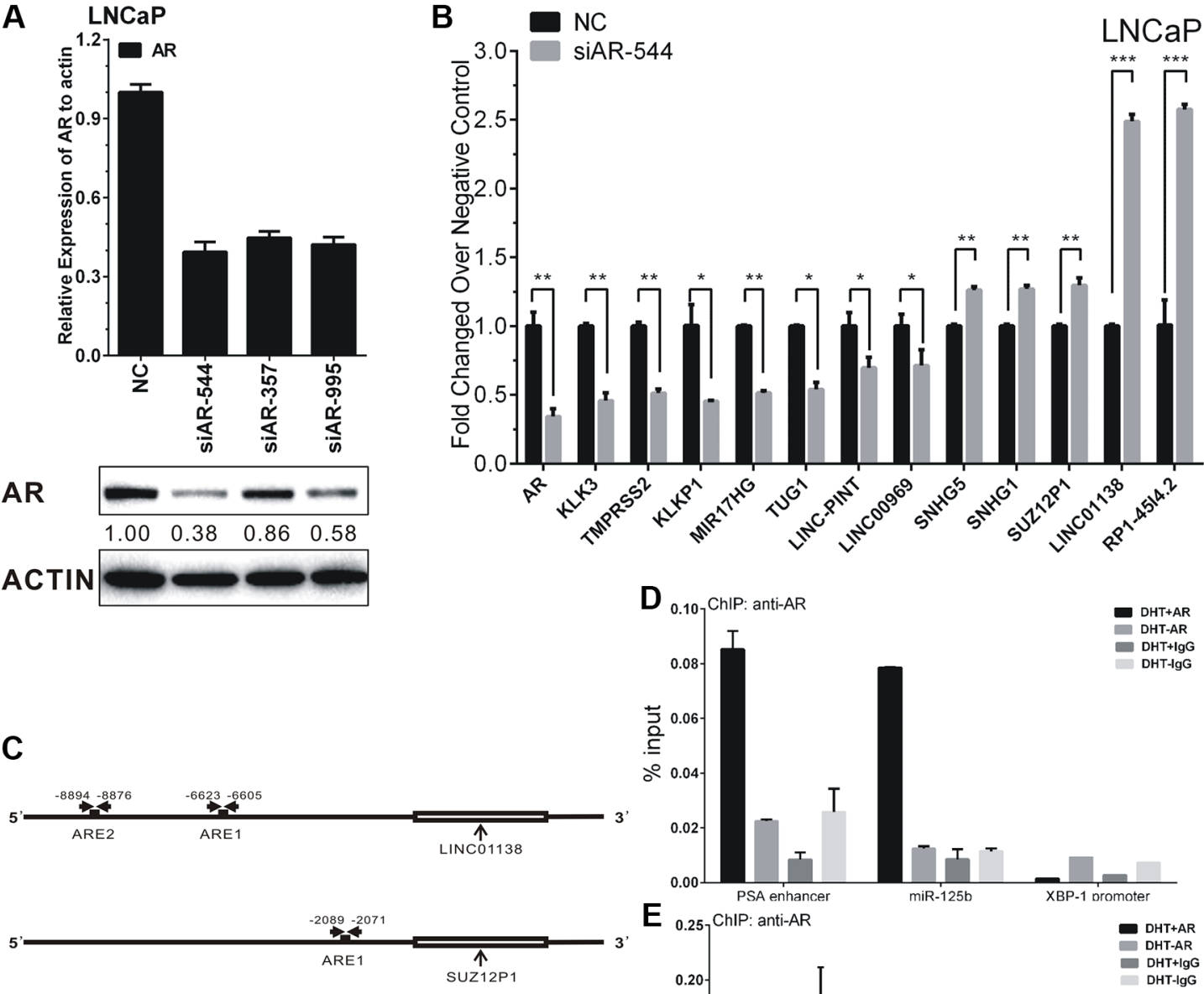

E
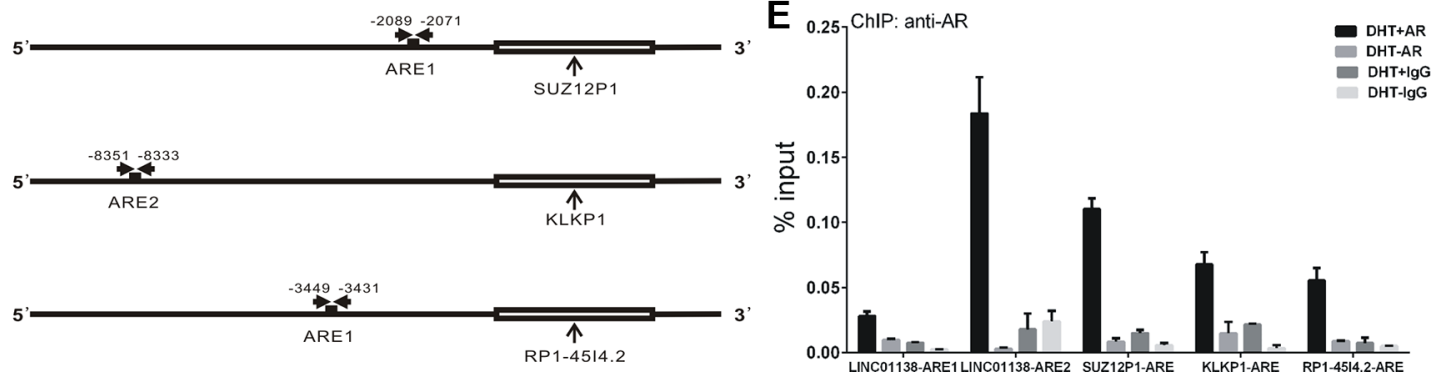

LINC01138-ARE1 LINC01138-ARE2 SUZ12P1-ARE KLKP1-ARE RP1-45I4.2-ARE

Figure 4: AR's regulation on IncRNAs' expression in LNCaP cells. (A) The efficiency of siAR-544, siAR-357 and siAR-995 effecting on AR expression were confirmed by RT-PCR and western blot. Protein bands from Western blot assay were quantified using Quantity One software (Bio-Rad, USA). (B) Expressions of ten lncRNAs after transfection of siAR-544 compared with NC in LNCaP cells. (C) Schematic diagram of AREs of LINC01138, SUZ12P1, KLKP1 and RP1-45I4.2. The approximate ARE locations were indicated by the horizontal arrows. (D-E) ChIP assay of AR-binding on candidate AREs of LINC01138, SUZ12P1, KLKP1 and RP1-45I4.2. ChIP assays of PSA enhancer (KLK3) and miR-125b ARE serve as positive control, and XBP-1 promoter serve as negative control for AR-binding. Values, expressed as percentages of input DNA, are presented as the mean $\pm \mathrm{SD}$ of at least three independent experiments. Significance was defined as $p<0.05\left({ }^{*} p<0.05 ;{ }^{*} p<0.01 ; * * * p<0.001\right)$. 
LNCaP cells lines (Figure 8G). The results revealed that LINC01138 and SUZ12P1 functioned as oncogenes in prostate cancer.

\section{DISCUSSION}

The molecular mechanisms involved in the development and androgen-resistance transformation of PCa remains unclear. Therefore, further study of castration-resistant $\mathrm{PCa}$ is of great importance. Recent studies indicate that $\operatorname{lncRNAs}$ play an important role in tumorigenesis and tumor metastasis [27-29]. In previous studies, several lncRNAs, including CTBP1-AS [6], PCAT18 [16], and PCAT29 [17], have been identified as targets of AR and were associated with PCa androgen-resistance transformation. Interestingly, NEAT1, an oestrogen receptor alpha-regulated lncRNA, promoted prostate tumorigenesis and was associated with therapeutic resistance as well [30].

In this study, we performed the dynamic microarray experiment for simultaneously observing expressions of genome-wide lncRNAs in LNCaP cells stimulated by DHT for $0 \mathrm{~h}$ and $2 \mathrm{~h}$, respectively. Supervised analysis of the microarray data showed a total of $3767 \mathrm{lncRNAs}$ (1991 up-regulated and 1776 down-regulated) produced from 2980 genes were differentially expressed after DHT stimulation. Combined with ChIPBase data [25], 312 lncRNAs overlapping AR peaks may be directly regulated by AR. As far as we know, this is the first study to identify androgen-responsive lncRNAs globally.

Another important topic is the exploration of prognostic markers for $\mathrm{PCa}$, especially for

A

A GSE55909
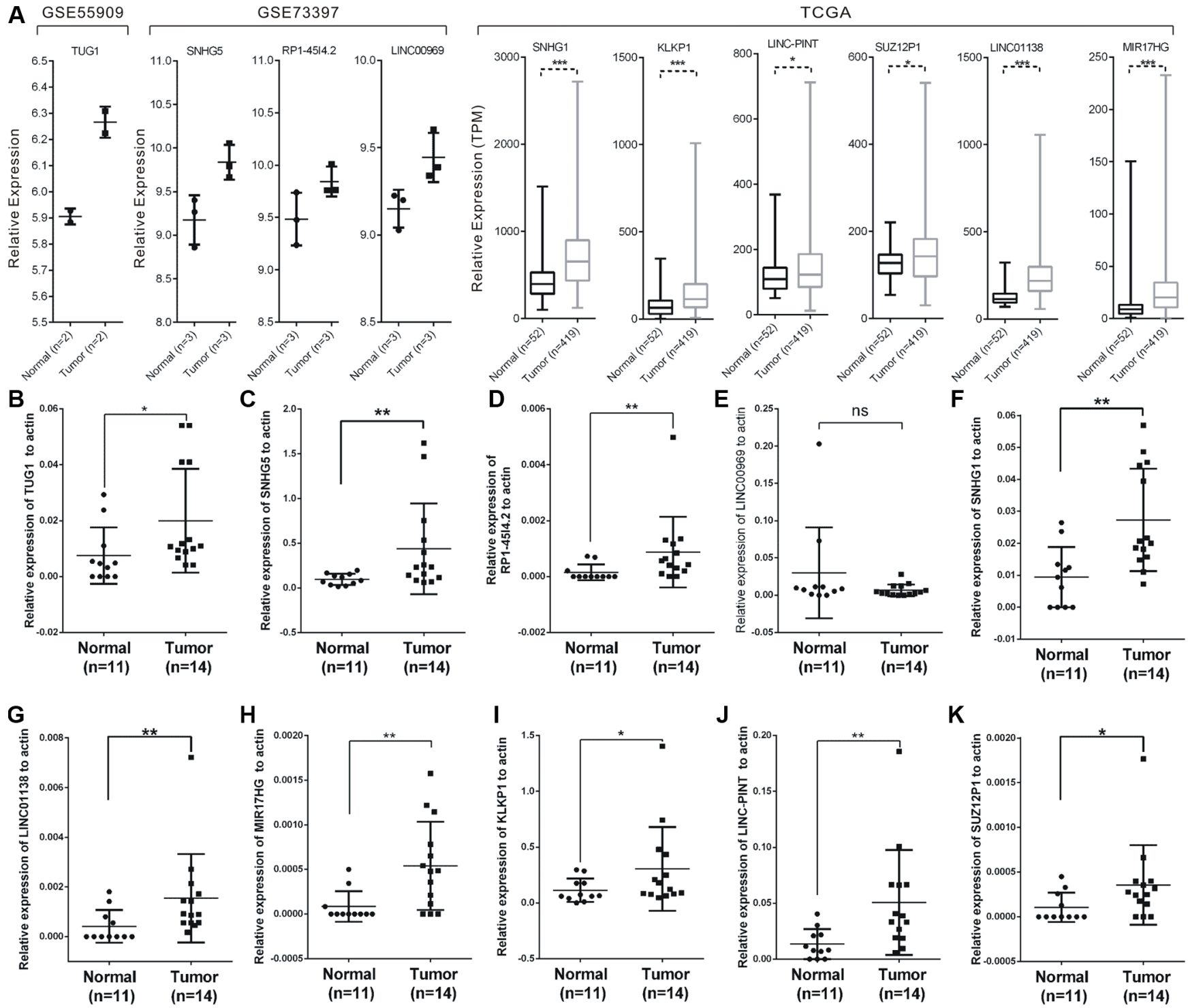

K

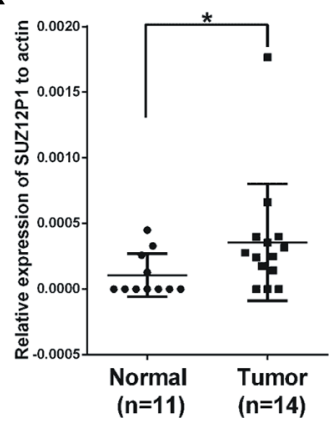

Figure 5: Expressions of candidate IncRNAs in tissue samples. The expression level of candidate lncRNAs from microarray data and TCGA data were shown (A). (B-K) The expression levels of ten lncRNAs in 11 normal prostate tissues and 14 prostate cancer tissues measured by RT-PCR. Significance was defined as $p<0.05(* p<0.05 ; * * p<0.01 ; * * * p 0.001)$. 
castration-resistant PCa. To identify novel diagnostic and prognostic markers, we firstly investigated lncRNAs expression signature of $\mathrm{PCa}$ samples from patients. Another two publicly available gene expression data, TCGA and GSE55909 were also used to identify the differentially expressed lncRNAs in prostate cancer. Three thousand and eighty-two lncRNA transcripts (934 up-regulated and 2148 down-regulated lncRNAs) were found to be significantly differentially expressed. Some studies showed that RNA-Seq methods (TCGA) and microarray-based methods did not have high coincidence degree while they both are good technologies to measure gene expression level [31]. In present study, only $12 \%$ of up-regulated and lncRNAs of TCGA data (31/252) were also identified to be overexpressed in microarray data, consistent with previous reports. To maximize the coverage of lncRNA expression profiling and hold more useful information for further study, we used the union of TCGA and microarray results instead overlap of TCGA and microarray results. Novel lncRNAs expression signatures were revealed in these tissues.

A total of 44 androgen-responsive lncRNAs were found to be over-expressed and 95 lncRNAs were found to be down-regulated in PCa samples (Figure 1D and 1E). Of 44 up-regulated androgen-responsive lncRNAs, 3 androgen-reduced lncRNAs and 7 androgen-induced lncRNAs were randomly selected for further study. Using qRT-PCR assay, we validated that RP1-4514.2, SUZ12P1, SNHG5, LINC01138, and SNHG1, were downregulated, and KLKP1, LINC00969, LINC-PINT, TUG1 and MIR17HG, were up-regulated after DHT stimulation in both time- and dose-dependent manner. Furthermore, we found AR knockdown could promote expressions of androgen- reduced lncRNAs and inhibit androgen- induced lncRNAs' expression. Based on these results, we identified AREs of RP1-4514.2, LINC01138, SUZ12P1 and KLKP1 using ChIP assay. As a result, a significant increase of AR binding to the chromatin of putative AREs in RP1-4514.2, LINC01138, SUZ12P1 and KLKP1, was showed in LNCaP cells treated with $100 \mathrm{nM}$ DHT for $4 \mathrm{~h}$. Moreover, qRT-PCR assay showed that these ten lncRNAs were significantly up-regulated in PCa tumors and cell lines.

To evaluated prognostic values of differentially expressed androgen-responsive lncRNAs in PCa,we then analyzed TCGA database and found that more than 90 transcripts were significantly differentially expressed in invasive extraprostatic tumors (pT3a , pT3b and T4 stages) compared with intraprostatic localized tumors (pT2a , pT2b and pT2c stages). Our study identified three androgen-responsive lncRNAs, LINC01138, SUZ12P1 and SNHG1 were significantly over-expressed in more metastasis and higher tumor stage patients. Furthermore, we conducted a comprehensive analysis to identify the correlation between androgen-responsive lncRNAs levels and biochemical recurrence, and found that a high level of LINC01138, SUZ12P1 and SNHG1 expression was associated with high biochemical recurrence. Moreover, we found that knockdown of LINC01138 and SUZ12P1 significantly inhibited cell proliferation and promoted cell apoptosis. These results suggested that these lncRNAs

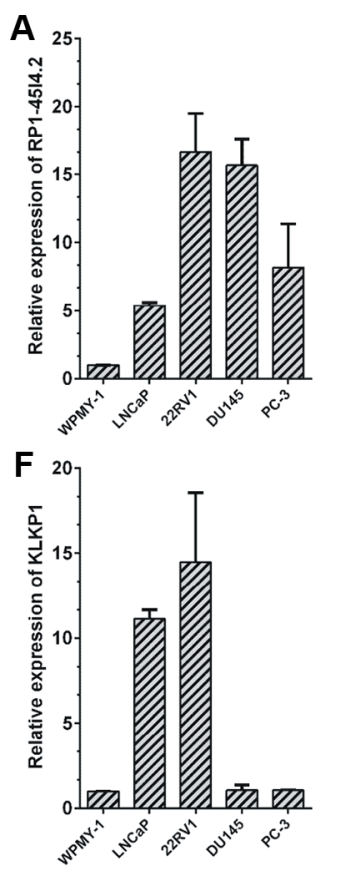

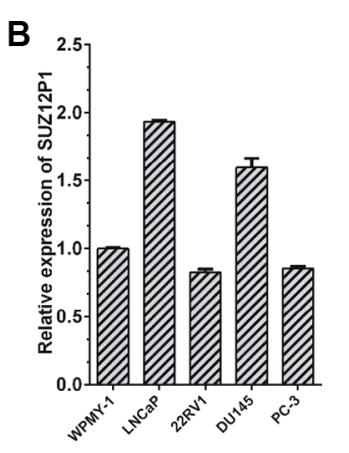

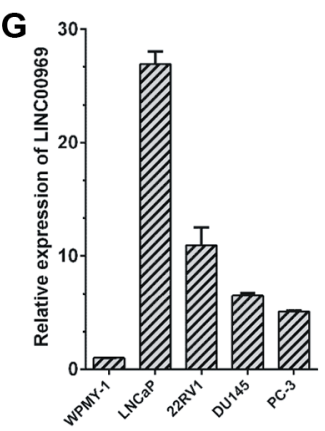

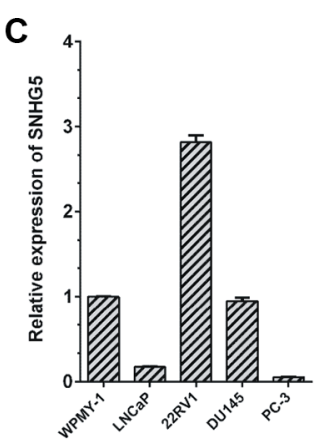

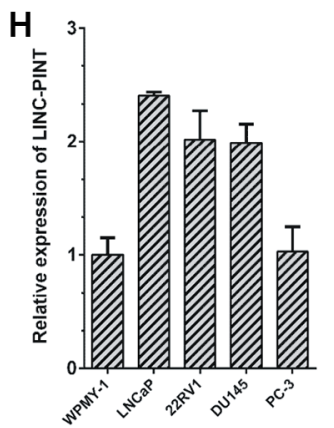

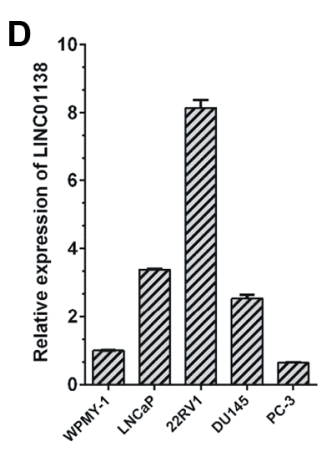
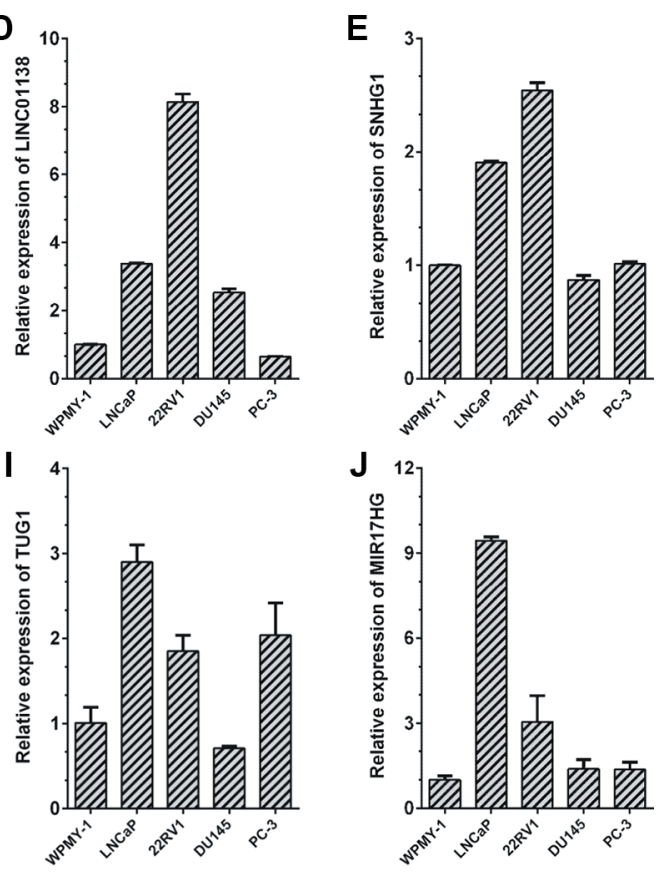

Figure 6: Expressions of candidate IncRNAs in prostate cancer cell lines. qRT-PCR analysis of ten lncRNAs' expression levels in normal prostate epithelial cells line WPMY-1 and four prostate cancer cell lines, including LNCaP, 22Rv1, DU145 and PC-3. The $\mathrm{Ct}$ value of each lncRNA was normalized to the $\mathrm{Ct}$ value of $\beta$-actin, and the relative expression was calculated by comparing with WPMY-1 cells by calculating the $2^{-\Delta \Delta \mathrm{Ct}}$ method. Each sample was run in triplicate to ensure quantitative accuracy. 
may act as oncogenes. Puzzlingly, all of the three lncRNAs were androgen-reduced genes, indicating that the expression of LINC01138, SUZ12P1 and SNHG1 may be repressed by AR activity in "androgen-dependent" tumors. There may be other pathways regulating these lncRNAs expression in PCa.
Several limitations of this study should be noted. First, the time points of time-course microarray is limited, and that make it restrict to observe dynamic expression of lncRNAs. Second, due to the limited sample size of data in the present study, further studies based on larger series of patients are needed to confirm the significance of the
A

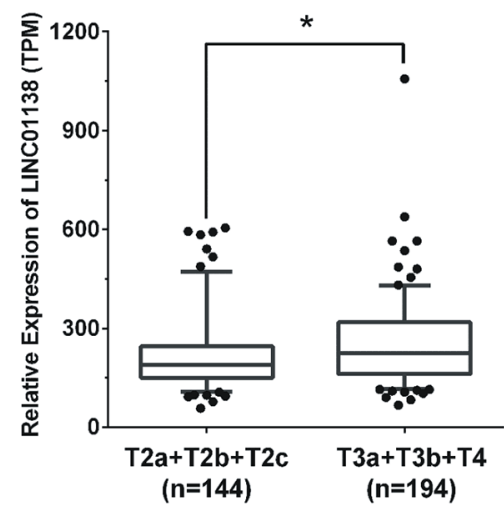

Pathological stage

D

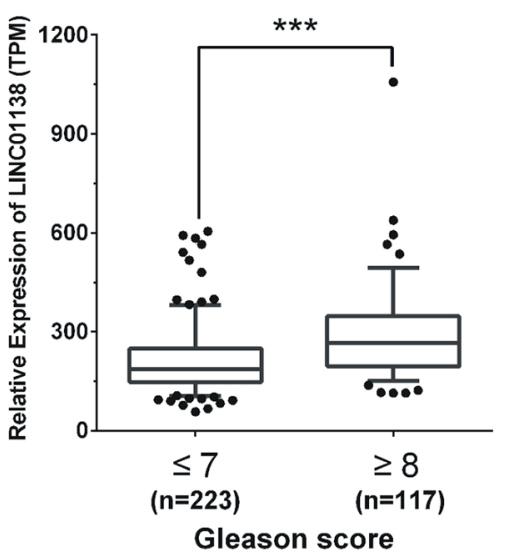

G

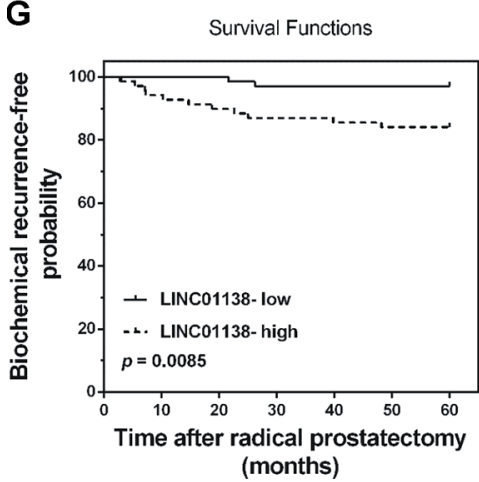

B

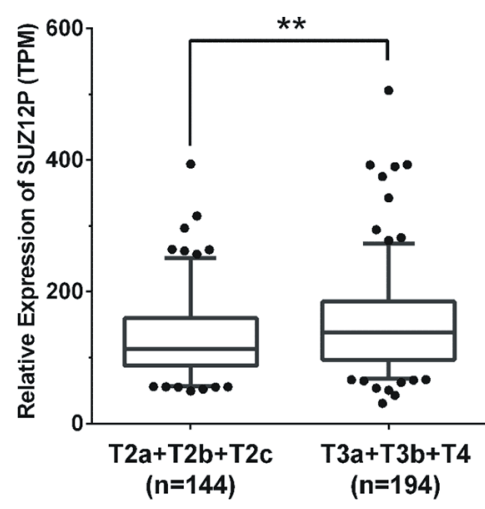

Pathological stage

E

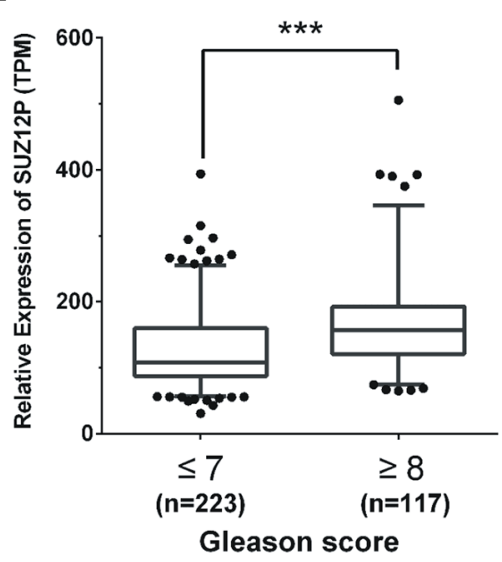

H

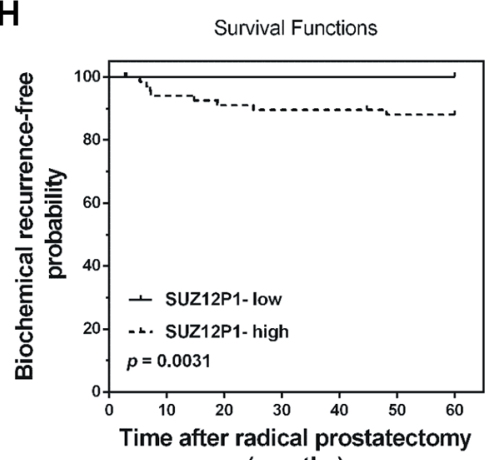

(months)
C

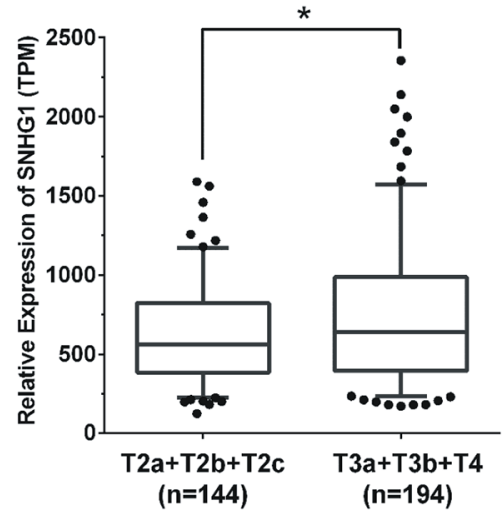

Pathological stage

$\mathbf{F}$

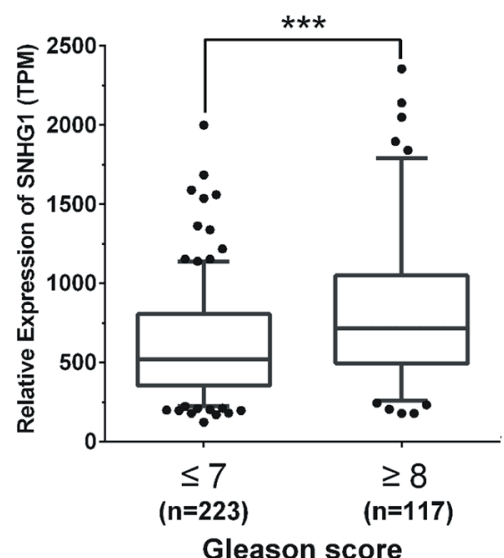

I

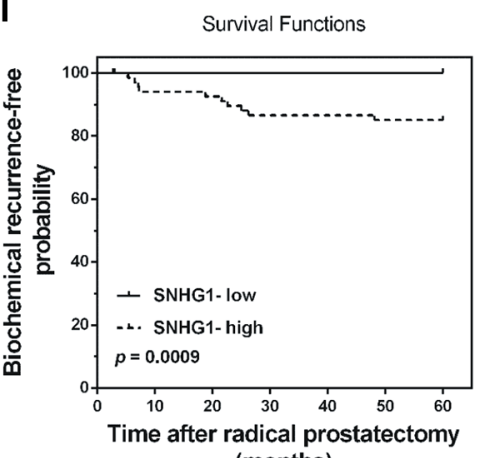

(months)

Figure 7: Pathological analyses of LINC01138, SUZ12P1 and SNHG1. (A-C) High expression levels of LINC01138, SUZ12P1 and SNHG1 in invasive extraprostatic tumors (pT3a, pT3b and T4 stages) compared with intraprostatic localized tumors (pT2a, pT2b and pT2c stages) according to publicly available gene expression data TCGA. (D-F) Low expression levels of LINC01138, SUZ12P1 and SNHG1 expression levels in prostate tumors with a low Gleason score $(\leq 7)$ compared with tumors with Gleason score $\geq 8$ according to publicly available gene expression data TCGA. (G-I) Kaplan-Meier curves for survival time after radical prostatectomy in patients with prostate cancer according to expression of LINC01138, SUZ12P1 and SNHG1. Significance was defined as $p<0.05\left({ }^{*} p<0.05 ; * * p<0.01\right.$; $* * * p<0.001)$. 

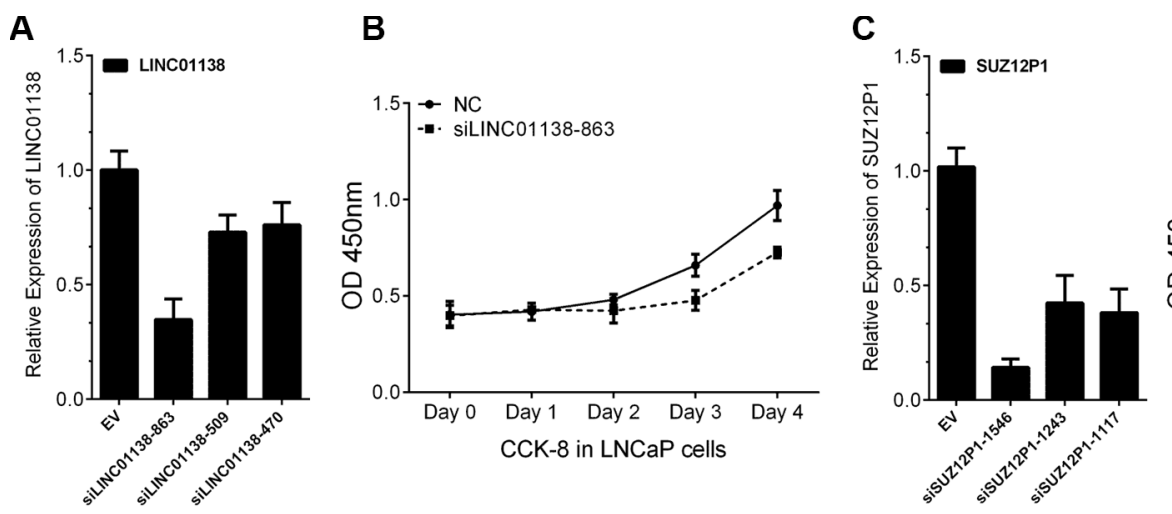

D
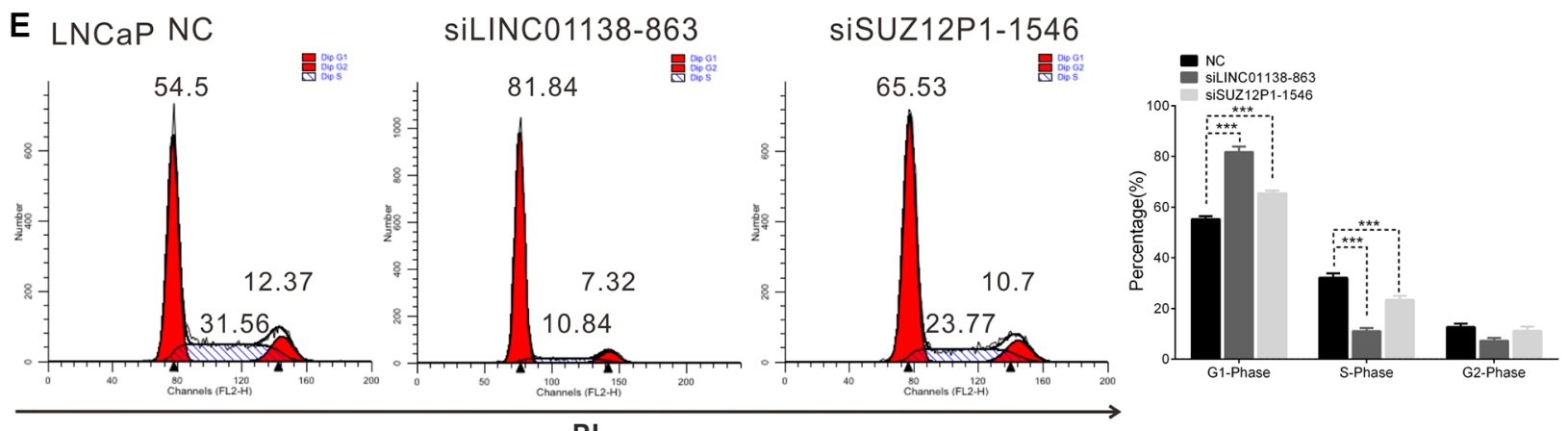

F $P C-3 \quad N C$

PI
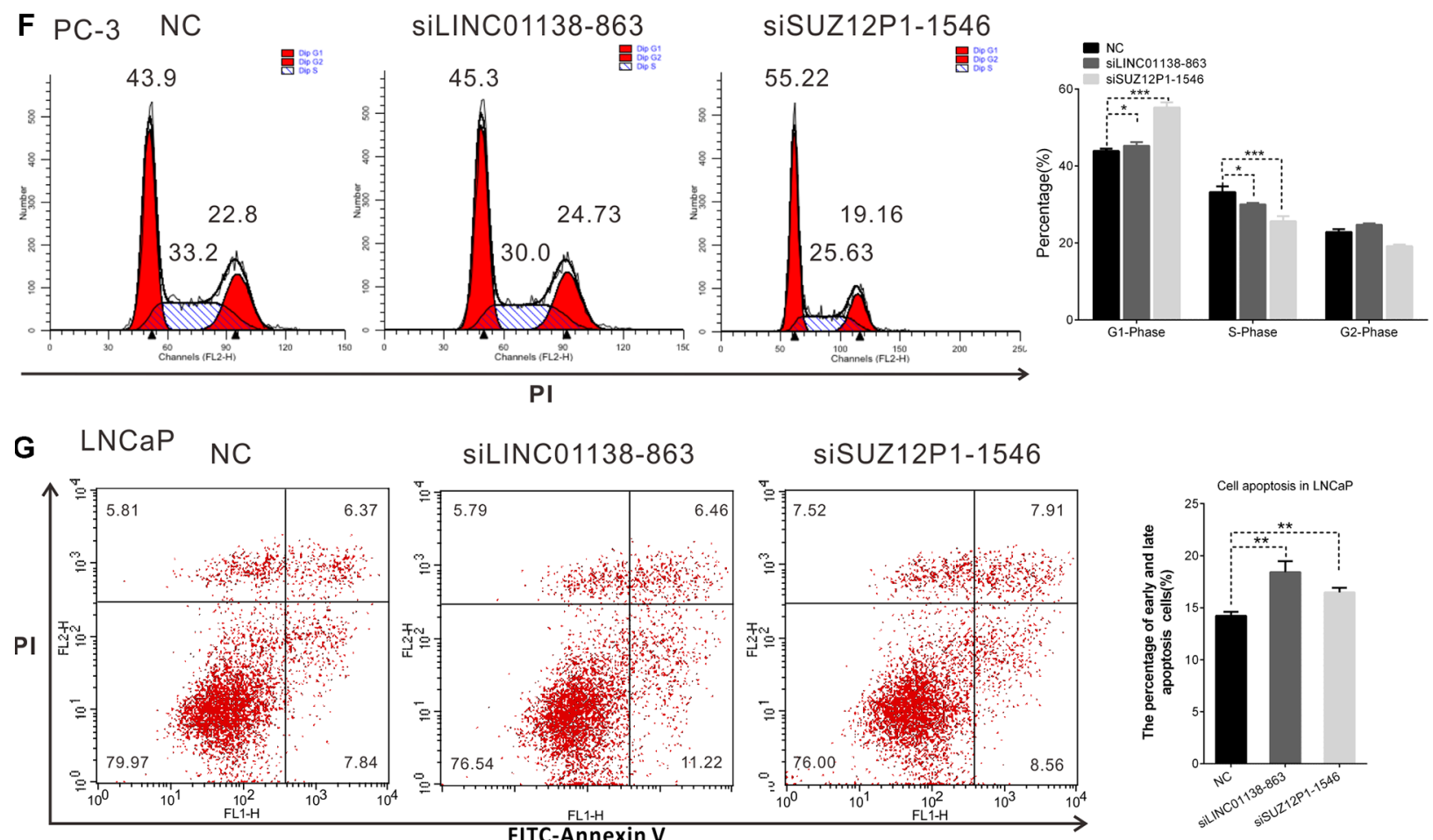

Figure 8: LINC01138 and SUZ12P1 promotes cell proliferation, cell cycle and inhibits apoptosis of PCa. (A and C) The efficiency of siRNAs effecting on LINC01138 and SUZ12P1 expression were confirmed by RT-PCR. (B and D) LINC01138 and SUZ12P1 knockdown significantly inhibit LNCaP cell proliferation. (E, F) Cell cycle assay was performed in LNCaP and PC-3 cells. Cells were transfected with siLINC01138 or siSUZ12P1 for $48 \mathrm{~h}$, stained with PI and evaluated with a FACScalibur flow cytometer. LINC01138 and SUZ12P1 knockdown inhibit cell cycle progression in LNCaP and PC-3 cells. (G) Cell apoptosis assay was performed with flow cytometer. Cells were transfected with siLINC01138 or siSUZ12P1 for $48 \mathrm{~h}$, and subjected to cell apoptosis assay. LINC01138 and SUZ12P1 knockdown in LNCaP cells increased the fraction of both early apoptotic cells and late apoptotic cells. The cell cycle and apoptosis analysis results presented as mean $\pm \mathrm{SD}(n=3)$. Significance was defined as $p<0.05\left({ }^{*} p<0.05 ;{ }^{* *} p<0.01 ;{ }^{* * *} p<0.001\right)$; ns means not significant. 
signature. Last, additional function investigations of these IncRNAs on PCa are still needed.

In conclusion, our study represents the comprehensive analysis of androgen-responsive lncRNAs in prostate cancer for the first time. Analyzing the expression of these lncRNAs is likely to provide novel therapeutic targets for $\mathrm{PCa}$, counting the general of the recently approved therapies for mCRPC targeting the AR pathway $[16,32]$. We also analyzed the expression patterns of lncRNAs in PCa samples, and identified a set of IncRNAs that are aberrantly expressed between prostate cancers and normal controls as well as the aberrant expressed lncRNAs between invasive extraprostatic tumors and intraprostatic localized tumors. These data suggest that these lncRNAs may be used as potential prognostic targets and predict patient outcome. We believe this study provides useful information for exploring potential therapeutic and prognostic targets for prostate cancer, especially for castration-resistant $\mathrm{PCa}$.

\section{MATERIALS AND METHODS}

\section{Cell culture and androgen treatment}

LNCaP cells were purchased from the American Type Culture Collection (Manassas, USA) which was confirmed by short tandem repeat (STR) analysis. 22RV1, DU145, PC-3 and WPMY-1 were obtained from Cell Bank of Chinese Academy of Sciences (Shanghai, China) where they were authenticated by mycoplasma detection, DNA-Fingerprinting, isozyme detection and cell vitality detection. All experiments were carried out by each cell line at passages below 30 . The four prostate cancer cell lines were maintained in RPMI 1640 medium (Corning, USA) supplemented with 10\% FBS (Hyclone, USA) and WPMY-1 in DMEM medium (Corning, USA) with $10 \%$ FBS, and they all cultured at $37^{\circ} \mathrm{C}$ in $5 \% \mathrm{CO} 2$.

Androgen treatment assay was performed as described previously [33]. Briefly, LNCaP cells were cultivated in Phenol Red-free RPMI 1640 (GIBCO/BRL) supplemented with $10 \%$ charcoal-dextran-stripped FBS for 3 days before androgen treatment, then were induced with DHT at concentration of $10 \mathrm{nM}$. The genome-wide dynamic response to DHT was analyzed at five time points $-0 \mathrm{~h}, 2 \mathrm{~h}, 8 \mathrm{~h}, 24 \mathrm{~h}$ and $48 \mathrm{~h}$, where ' $0 \mathrm{~h}$ ' represents the state before androgen action.

\section{Tissue collection}

The trial was approved by the Research Ethics Committee of Tongji Hospital and verbal consent was obtained from all patients. Three tumor tissues and three adjacent normal tissues were used for microarray analysis of lncRNAs. Fourteen tumor tissues and 11 adjacent normal tissues were used for an extra evaluation by qRT-PCR. All samples were collected from Tongji Hospital, a subsidiary of Shanghai Tongji University, between January
2001 and December 2013. The prostate cancer patients whom the tissues were obtained from underwent radical prostatectomy and did not receive any pre-operation treatment. The histopathological features of tumor specimens were classified according to the Gleason score system and 2002 TNM classification system.

\section{Microarray and expression data sets}

Total RNA of samples was isolated by using TRIzol (Invitrogen) and the RNeasy mini kit (QIAGEN). Total RNA was quantified by the NanoDrop ND-2000 (Thermo Scientific) and the RNA integrity was assessed using Agilent Bioanalyzer 2100 (Agilent Technologies).

Total RNA from LNCaP cells, treated with $10 \mathrm{nM}$ DHT for 0 and $2 \mathrm{hr}$, were hybridized to Agilent Human IncRNA (4*180K, Design ID: 042818). The sample labeling, microarray hybridization and washing were performed based on the manufacturer's standard protocols. Briefly, total RNA were transcribed to double strand cDNA, then synthesized into cRNA and labeled with Cyanine-3-CTP. The labeled cRNAs were hybridized onto the microarray. After washing, the arrays were scanned by the Agilent Scanner G2505C (Agilent Technologies). Feature Extraction software (version10.7.1.1, Agilent Technologies) was used to analyze array images to get raw data. Genespring were employed to finish the basic analysis with the raw data. The raw microarray data were uploaded to the Gene Expression Omnibus public repository (http://www.ncbi.nlm.nih.gov/geo/query/acc. cgi? acc = GSE72866; Gene Expression Omnibus series no. GSE72866). To begin with, the raw data was normalized with the quantile algorithm. The probes that at least 1 conditions out of 2 conditions have flags in "P" were chosen for further data analysis. Differentially expressed lncRNAs were then identified through fold change. The threshold set for up- and down-regulated genes was a fold change $>=2.0$.

Global expressions of lncRNAs in 3 PCa samples and 3 normal prostate tissues were examined microarray version A10312-90-2 (RiboBio), containing 41532 lncRNA probes and 33352 mRNA probes. The sample labeling, microarray hybridization and washing were performed based on the manufacturer's standard protocols. The raw microarray data were uploaded to the Gene Expression Omnibus public repository (http://www.ncbi.nlm.nih.gov/ geo/; Gene Expression Omnibus series no. GSE73397). Raw data was normalized with the $\log 2$ scale. Two-class unpaired significance analysis of microarray (SAM) [34] was employed to filter significantly differentially expressed lncRNA between PCa and normal samples (Reference). Following 1000 permutations, LncRNAs were selected with discriminating parameter of $q<0.05$.

In this paper, only lncRNAs with description from NCBI or Ensemble were selected for further study.

Another two publicly available gene expression data, TCGA Data Portal (https://tcga-data.nci.nih.gov/ 
tcga/) and GSE55909 [35], were also used to identify the differentially expressed lncRNAs in Prostate Cancer.

\section{RNA interference and transient transfection}

All siRNA oligonucleotides against AR and negative control (NC) were were purchased from GenePharma (Shanghai, China), and used at $50 \mathrm{nM}$ concentration. All sequences of synthetic oligonucleotides are listed in Supplementary Table S6. Transfection was carried out with Lipofectamine 2000 Transfection Reagent (Life, USA) according to the manufacturer's procedure.

\section{Real-time reverse transcription PCR (qRT-PCR) analysis}

qRT-PCR for lncRNAs and mRNAs was performed using AceQ qPCR SYBR Green Master Mix (Vazyme Biotech co., ltd) [36]. Primers used for qRT-PCR were listed in Supplementary Table S6. The Ct values were normalized using $\beta$-actin as internal control to estimate the different expression of genes. Relative mRNA expression was calculated using the $2^{-\Delta \Delta \mathrm{Ct}}$ method. Each sample was run in triplicate to ensure quantitative accuracy.

\section{Chromatin immunoprecipitation (ChIP) assay}

ChIP was performed as described previously [37]. Chromatin immunoprecipitates for proteins and methyl marks were amplified by quantitative PCR, normalized to input, and calculated as percentages of inputs. Fold enrichment levels indicate the fold changes over the negative control immunoglobulin $\mathrm{G}(\mathrm{IgG})$. The primers for qRT-PCR analysis of DNA fragments containing ARE were listed in Supplementary Table S6, particularly KLK3 (PSA) enhancer and miR-125b ARE work as the positive control, whereas XBP-1 promoter works as the negative control. A DNA region without a putative ARE also served as negative control.

\section{Cell proliferation assay}

Cell proliferation analysis was performed with Cell Counting Kit-8 (CCK-8, Dojindo Laboratories, Kumamoto, Japan) in octuplicate according to the manufacturer's instructions. Briefly, cells of 5000 per well were seeded into 96-well plate, and examined at the time point of $0,24,48,72,96$, and $120 \mathrm{~h}$. At each time point, CCK-8 (10 $\mu \mathrm{l})$ was added to the wells, and after an incubation of $2 \mathrm{~h}$ at $37^{\circ} \mathrm{C}$, absorbance was measured at $450 \mathrm{~nm}$ with a Microplate Reader ELx808 (Bio-Tek, VT, USA).

\section{Cell cycle and apoptosis assay}

Cells were harvested $48 \mathrm{~h}$ after transfection. For cycle assay, cells were incubated with $0.03 \%$ triton
$\mathrm{X}-100$ and propidium iodide (PI) $(50 \mathrm{ng} / \mathrm{mL})$ for $15 \mathrm{~min}$; the percentages of cells in different phases of cell cycle were measured with a FACScalibur flow cytometer (BD, CA, USA) and analyzed with ModFit software (Verity Software House, ME, USA). For apoptosis assay, cells were assayed with FITC Annexin V Apoptosis Detection Kit (BD, CA, USA) and analyzed by flow cytometry.

\section{Western blotting analysis}

Western blotting was performed as described previously using antibodies against AR (Millipore) and actin (Sigma) [36]. The protein concentration was determined by the BCA Protein Assay Kit (Novoprotein Scientific Inc., China), in accordance with the manufacturer's instructions. Signal intensity of Western blots was quantified by Quantity One Software (Bio-Rad, USA).

\section{Statistical analysis}

The numerical data were presented as mean \pm standard deviation (SD) of at least three determinations. Statistical comparisons between groups of normalized data were performed using $T$-test or Mann-Whitney $U$-test according to the test condition. A $p<0.05$ was considered statistical significance with a $95 \%$ confidence level.

\section{ACKNOWLEDGMENTS AND FUNDING}

This work is supported by a grant 31571330 from the National Natural Science Foundation of China, a grant 13DZ2252000 by Shanghai Science and Technology Commission, and Project SKLGE-1403 supported by the Research Fund of the State Key Laboratory of Genetic Engineering, Fudan University.

\section{CONFLICTS OF INTEREST}

The authors declare no financial conflicts of interest.

\section{REFERENCES}

1. Kornienko AE, Guenzl PM, Barlow DP, Pauler FM. Gene regulation by the act of long non-coding RNA transcription. BMC biology. 2013; 11:59.

2. Song W, Liu YY, Peng JJ, Liang HH, Chen HY, Chen JH, He WL, Xu JB, Cai SR, He YL. Identification of differentially expressed signatures of long non-coding RNAs associated with different metastatic potentials in gastric cancer. J Gastroenterol. 2015.

3. Sun M, Xia R, Jin FY, Xu TP, Liu ZJ, De W, Liu XH. Downregulated long noncoding RNA MEG3 is associated with poor prognosis and promotes cell proliferation in gastric cancer. Tumor Biol. 2014; 35:1065-1073.

4. Kim T, Cui R, Jeon YJ, Fadda P, Alder H, Croce CM. MYCrepressed long noncoding RNAs antagonize MYC-induced 
cell proliferation and cell cycle progression. Oncotarget. 2015; 6:18780-18789.

5. Geisler S, Coller J. RNA in unexpected places: long noncoding RNA functions in diverse cellular contexts. Nat Rev Mol Cell Bio. 2013; 14:699-712.

6. Takayama K, Horie-Inoue K, Katayama S, Suzuki T, Tsutsumi S, Ikeda K, Urano T, Fujimura T, Takagi K, Takahashi S, Homma Y, Ouchi Y, Aburatani H, et al. Androgen-responsive long noncoding RNA CTBP1-AS promotes prostate cancer. Embo J. 2013; 32:1665-1680.

7. Yang LQ, Lin CR, Jin CY, Yang JC, Tanasa B, Li WB, Merkurjev D, Ohgi KA, Meng D, Zhang J, Evans CP, Rosenfeld MG. IncRNA-dependent mechanisms of androgen-receptor-regulated gene activation programs. Nature. 2013; 500:598-602.

8. Pickard MR, Williams GT. Molecular and Cellular Mechanisms of Action of Tumour Suppressor GAS5 LncRNA. Genes. 2015; 6:484-499.

9. Huarte M, Guttman M, Feldser D, Garber M, Koziol MJ, Kenzelmann-Broz D, Khalil AM, Zuk O, Amit I, Rabani M, Attardi LD, Regev A, Lander ES, et al. A large intergenic noncoding RNA induced by p53 mediates global gene repression in the p53 response. Cell. 2010; 142:409-419.

10. Malik R, Patel L, Prensner JR, Shi Y, Iyer MK, Subramaniyan S, Carley A, Niknafs YS, Sahu A, Han S, Ma T, Liu M, Asangani IA, et al. The lncRNA PCAT29 inhibits oncogenic phenotypes in prostate cancer. Mol Cancer Biol. 2014; 12:1081-1087.

11. Siegel RL, Miller KD, Jemal A. Cancer statistics, 2016. CA Cancer J Clin. 2016; 66:7-30.

12. Hara T, Miyazaki H, Lee A, Tran CP, Reiter RE. Androgen receptor and invasion in prostate cancer. Cancer research. $2008 ; 68: 1128-1135$.

13. Wang Q, Li W, Zhang Y, Yuan X, Xu K, Yu J, Chen Z, Beroukhim R, Wang H, Lupien M, Wu T, Regan MM, Meyer CA, et al. Androgen receptor regulates a distinct transcription program in androgen-independent prostate cancer. Cell. 2009; 138:245-256.

14. Small EJ, Reese DM, Vogelzang NJ. Hormone-refractory prostate cancer: an evolving standard of care. Semin Oncol. 1999; 26:61-67. doi: 10.18632/oncotarget.3909.

15. Locke JA, Guns ES, Lubik AA, Adomat HH, Hendy SC, Wood CA, Ettinger SL, Gleave ME, Nelson CC. Androgen levels increase by intratumoral de novo steroidogenesis during progression of castration-resistant prostate cancer. Cancer research. 2008; 68:6407-6415.

16. Crea F, Watahiki A, Quagliata L, Xue H, Pikor L, Parolia A, Wang YW, Lin D, Lam WL, Farrar WL, Isogai T, Morant R, Castori-Eppenberger $\mathrm{S}$, et al. Identification of a long noncoding RNA as a novel biomarker and potential therapeutic target for metastatic prostate cancer. Oncotarget. 2014; 5:764-774. doi: 10.18632/oncotarget.1769.

17. Malik R, Patel L, Prensner JR, Shi Y, Iyer MK, Subramaniyan S, Carley A, Niknafs YS, Sahu A, Han SM,
Ma T, Liu ML, Asangani IA, et al. The lncRNA PCAT29 Inhibits Oncogenic Phenotypes in Prostate Cancer. Mol Cancer Biol. 2014; 12:1081-1087.

18. Prensner JR, Rubin MA, Wei JT, Chinnaiyan AM. Beyond PSA: The Next Generation of Prostate Cancer Biomarkers. Sci Transl Med. 2012; 4.

19. Bottcher R, Hoogland AM, Dits N, Verhoef EI, Kweldam C, Waranecki P, Bangma CH, van Leenders GJLH, Jenster G. Novel long non-coding RNAs are specific diagnostic and prognostic markers for prostate cancer. Oncotarget. 2015; 6:4036-4050. doi: 10.18632/oncotarget.2879.

20. Mehra R, Shi Y, Udager AM, Prensner JR, Sahu A, Iyer MK, Siddiqui J, Cao XH, Wei J, Jiang H, Feng FY, Chinnaiyan AM. A Novel RNA In Situ Hybridization Assay for the Long Noncoding RNA SChLAP1 Predicts Poor Clinical Outcome After Radical Prostatectomy in Clinically Localized Prostate Cancer. Neoplasia. 2014; 16:1121-1127.

21. Srikantan V, Zou ZQ, Petrovics G, Xu L, Augustus M, Davis L, Livezey JK, Connell T, Sesterhenn IA, Yoshino K, Buzard GS, Mostofi FK, McLeod DG, et al. PCGEM1, a prostate-specific gene, is overexpressed in prostate cancer. Proc Natl Acad Sci USA. 2000; 97:12216-12221.

22. Bussemakers MJG, van Bokhoven A, Verhaegh GW, Smit FP, Karthaus HFM, Schalken JA, Debruyne FMJ, $\mathrm{Ru} \mathrm{N}$, Issacs WB. DD3: A new prostate-specific gene, highly overexpressed in prostate cancer. Cancer research. 1999; 59:5975-5979.

23. Dijkstra S, Mulders PFA, Schalken JA. Clinical use of novel urine and blo3od based prostate cancer biomarkers: A review. Clin Biochem. 2014; 47:889-896.

24. Leyten GHJM, Hessels D, Jannink SA, Smit FP, de Jong H, Cornel EB, de Reijke TM, Vergunst H, Kil P, Knipscheer BC, van Oort IM, Mulders PFA, Hulsbergenvan de Kaa CA, et al. Prospective Multicentre Evaluation of PCA3 and TMPRSS2-ERG Gene Fusions as Diagnostic and Prognostic Urinary Biomarkers for Prostate Cancer. European urology. 2014; 65:534-542.

25. Yang JH, Li JH, Jiang S, Zhou H, Qu LH. ChIPBase: a database for decoding the transcriptional regulation of long non-coding RNA and microRNA genes from ChIP-Seq data. Nucleic acids research. 2013; 41:D177-D187.

26. Cartharius K, Frech K, Grote K, Klocke B, Haltmeier M, Klingenhoff A, Frisch M, Bayerlein M, Werner $T$. MatInspector and beyond: promoter analysis based on transcription factor binding sites. Bioinformatics. 2005; 21:2933-2942.

27. Martens-Uzunova ES, Bottcher R, Croce CM, Jenster G, Visakorpi T, Calin GA. Long Noncoding RNA in Prostate, Bladder, and Kidney Cancer. European urology. 2014; 65:1140-1151.

28. Nagano T, Fraser P. No-Nonsense Functions for Long Noncoding RNAs. Cell. 2011; 145:178-181.

29. Prensner JR, Chinnaiyan AM. The Emergence of lncRNAs in Cancer Biology. Cancer Discov. 2011; 1:391-407. 
30. Chakravarty D, Sboner A, Nair SS, Giannopoulou E, Li RH, Hennig S, Mosquera JM, Pauwels J, Park K, Kossai M, MacDonald TY, Fontugne J, Erho N, et al. The oestrogen receptor alpha-regulated lncRNA NEAT1 is a critical modulator of prostate cancer. Nat Commun. 2014; 5.

31. Nazarov PV, Kaoma T, Muller A, Fritah S, Vallar L. Long Non-Coding RNAs in Lung Cancer: Comparison of Microarray and RNA-Seq Techniques. F1000Posters. 2013; 4:1446 (poster).

32. Bishr M, Saad F. Overview of the latest treatments for castration-resistant prostate cancer. Nat Rev Urol. 2013; 10:522-528.

33. Li X, Wan XC, Chen HB, Yang S, Liu YY, Mo WJ, Meng DL, Du WT, Huang Y, Wu H, Wang JQ, Li T, Li Y. Identification of miR-133b and RB1CC1 as Independent Predictors for Biochemical Recurrence and Potential Therapeutic Targets for Prostate Cancer. Clin Cancer Res. 2014; 20:2312-2325.

34. Tusher VG, Tibshirani R, Chu G. Significance analysis of microarrays applied to the ionizing radiation response. Proc Natl Acad Sci USA. 2001; 98:5116-5121.
35. Lee B, Mazar J, Aftab MN, Qi F, Shelley J, Li JL, Govindarajan S, Valerio F, Rivera I, Thurn T, Tran TA, Kameh D, Patel V, et al. Long Noncoding RNAs as Putative Biomarkers for Prostate Cancer Detection. J Mol Diagn. 2014; 16:615-626.

36. Mo WJ, Zhang JY, Li X, Meng DL, Gao Y, Yang S, Wan $\mathrm{XC}$, Zhou $\mathrm{CH}$, Guo FH, Huang Y, Amente S, Avvedimento EV, Xie Y, et al. Identification of Novel AR-Targeted MicroRNAs Mediating Androgen Signalling through Critical Pathways to Regulate Cell Viability in Prostate Cancer. PloS one. 2013; 8.

37. Yang S, Zhang JY, Zhang YL, Wan XC, Zhang CZ, Huang XH, Huang WH, Pu HL, Pei CH, Wu H, Huang Y, Huang SD, Li Y. KDM1A Triggers Androgen-Induced miRNA Transcription via H3K4me2 Demethylation and DNA Oxidation. Prostate. 2015; 75:936-946. 